\title{
An ALMA survey of the SCUBA-2 Cosmology Legacy Survey UKIDSS/UDS field: high-resolution dust continuum morphologies and the link between sub-millimetre galaxies and spheroid formation
}

\author{
B. Gullberg, ${ }^{1 \star}$ Ian Smail ${ }^{\oplus},{ }^{1}$ A. M. Swinbank ${ }^{\oplus},{ }^{1}$ U. Dudzevičiūtė ${ }^{\odot},{ }^{1}$ S. M. Stach, ${ }^{1}$ \\ A. P. Thomson, ${ }^{2}$ O. Almaini ${ }^{\oplus},{ }^{3}$ C. C. Chen ${ }^{\oplus},{ }^{4}$ C. Conselice,${ }^{3}$ E. A. Cooke ${ }^{\oplus}, 1$ \\ D. Farrah, ${ }^{5,6}$ R. J. Ivison ${ }^{\odot},{ }^{4,7}$ D. Maltby, ${ }^{3}$ M. J. Michałowski ${ }^{\oplus},{ }^{8}$ J. M. Simpson, ${ }^{9}$ \\ D. Scott, ${ }^{10}$ J. L. Wardlow ${ }^{1,11}$ and A. Weiss ${ }^{12}$ \\ ${ }^{1}$ Centre for Extragalactic Astronomy, Department of Physics, Durham University, South Road, Durham DH1 3LE, UK \\ ${ }^{2}$ Jodrell Bank Centre for Astrophysics, The University of Manchester, Oxford Road, Manchester M13 9PL, UK \\ ${ }^{3}$ School of Physics and Astronomy, University of Nottingham, University Park, Nottingham NG7 2RD, UK \\ ${ }^{4}$ European Southern Observatory, Karl-Schwarzschild-Straße 2, D-85748 Garching bei München, Germany \\ ${ }^{5}$ Department of Physics and Astronomy, University of Hawaii, 2505 Correa Road, Honolulu, HI 96822, USA \\ ${ }^{6}$ Institute for Astronomy, 2680 Woodlawn Drive, University of Hawaii, Honolulu, HI 96822, USA \\ ${ }^{7}$ Institute for Astronomy, University of Edinburgh, Royal Observatory, Blackford Hill, Edinburgh EH9 3HJ, UK \\ ${ }^{8}$ Astronomical Observatory Institute, Faculty of Physics, Adam Mickiewicz University, ul. Sloneczna 36, PL-60-286 Poznan, Poland \\ ${ }^{9}$ Academia Sinica Institute of Astronomy and Astrophysics, No. 1, Section 4, Roosevelt Road, Taipei 10617, Taiwan \\ ${ }^{10}$ Department of Physics and Astronomy, University of British Columbia, 6224 Agricultural Road, Vancouver, BC V6T 1Z1, Canada \\ ${ }^{11}$ Department of Physics, Lancaster University, Lancaster LA1 4YB, UK \\ ${ }^{12}$ Max-Planck-Institut fur Radioastronomie, Auf dem Hugel 69, D-53121 Bonn, Germany
}

Accepted 2019 September 30. Received 2019 September 30; in original form 2019 August 2

\begin{abstract}
We present an analysis of the morphology and profiles of the dust continuum emission in 153 bright sub-millimetre galaxies (SMGs) detected with ALMA at signal-to-noise ratios of $>8$ in high-resolution 0.18 arcsec $(\sim 1 \mathrm{kpc}) 870 \mu \mathrm{m}$ maps. We measure sizes, shapes, and light profiles for the rest-frame far-infrared emission from these luminous star-forming systems and derive a median effective radius $\left(R_{\mathrm{e}}\right)$ of $0.10 \pm 0.04$ arcsec for our sample with a median flux of $S_{870}=5.6 \pm 0.2 \mathrm{mJy}$. We find that the apparent axial ratio $(b / a)$ distribution of the SMGs peaks at $b / a \sim 0.63 \pm 0.02$ and is best described by triaxial morphologies, while their emission profiles are best fitted by a Sérsic model with $n \simeq 1.0 \pm 0.1$, similar to exponential discs. This combination of triaxiality and $n \sim 1$ Sérsic index are characteristic of bars and we suggest that the bulk of the $870 \mu \mathrm{m}$ dust continuum emission in the central $\sim 2 \mathrm{kpc}$ of these galaxies arises from bar-like structures. As such we caution against using the orientation of shape of the bright dust continuum emission at $\bar{\sim}$ resolution to assess either the orientation of any disc on the sky or tits inclination. By stacking our $870 \mu \mathrm{m}$ maps we recover faint extended dust continuum emission on $\sim 4 \mathrm{kpc}$ scales which contributes $13 \pm 1$ percent of the total $870 \mu \mathrm{m}$ emission. The scale of this extended emission is similar to that seen for the molecular gas and rest-frame optical light in these systems, suggesting that it represents an extended dust and gas disc at radii larger than the more active bar component. Including this component in our estimated size of the sources we derive a typical effective radius of $\simeq 0.15 \pm 0.05 \operatorname{arcsec}$ or $1.2 \pm 0.4 \mathrm{kpc}$. Our results suggest that kpc-scale bars are ubiquitous features of high starformation rate systems at $z \gg 1$, while these systems also contain fainter and more extended gas and stellar envelopes. We suggest that these features, seen some 10-12 Gyr ago, represent the formation phase of the earliest galactic-scale components: stellar bulges.
\end{abstract}

Key words: galaxies: evolution-galaxies: ISM-galaxies: starburst. 


\section{INTRODUCTION}

Sub-millimetre galaxies (SMGs) are a class of high-redshift dust obscured, but far-infrared luminous, galaxies with estimated starformation rates of $\sim 100-1000 \mathrm{M}_{\odot} \mathrm{yr}^{-1}$ (Smail, Ivison \& Blain 1997; Barger et al. 1998; Hughes et al. 1998).

The high star-formation rates are similar to those measured for local ultraluminous infrared galaxies (ULIRGs; e.g. Sanders \& Mirabel 1996; Tacconi et al. 2008; Engel et al. 2010; Riechers et al. 2011; Bothwell et al. 2013). The intense star-formation activity in local ULIRGs is believed to be triggered and fuelled by mergers, resulting in irregular morphologies at UV/optical wavelengths, with single and double nuclei and tidal tails (e.g. Clements \& Baker 1996; Farrah et al. 2001; Surace, Sanders \& Evans 2001; Veilleux 2002; Psychogyios et al. 2016). Theoretical models provide support for this suggestion: hydrodynamical simulations of mergers can result in remnants with a central starburst event building a bulge. After $\gtrsim 1$ Gyr the merger remnant comprises a central bulge with in situ star formation and an extended disc/envelope dominated by stars formed before the merger (Hopkins et al. 2013). It has been similarly suggested that major and minor mergers may also be the trigger for the activity in the high-redshift SMG population (e.g. McAlpine et al. 2019).

The spatial extents of local (U)LIRGs have been shown to vary strongly depending upon the observed wavelength: with the highest star-formation rate (U)LIRGs displaying the most extended emission in the optical, while at the same time showing the most compact emission in the mid-infrared, which is thought to trace the on-going star formation (Chen, Lowenthal \& Yun 2010; Psychogyios et al. 2016). Optical depths effects are a likely explanation for these varying trends, and this suggest that the physical size measured in the optical is highly dependent on the geometry of the dust distribution (Calzetti et al. 2007; Psychogyios et al. 2016). Comparisons of the rest-frame optical and far-infrared sizes of high-redshift SMGs have suggested similar behaviour, with much more extended optical sizes, compared to those derived from interferometric observations in the submillimetre, which trace the bulk of the star-formation activity visible in the rest-frame far-infrared waveband (Ikarashi et al. 2015; Simpson et al. 2015b; Hodge et al. 2016; Lutz et al. 2016).

However, while there are similarities, there are also apparent differences between the observed properties of SMGs and those of comparably strongly star-forming ULIRGs in the local Universe. One notable difference being the large spatial extent of the starformation activity in the high-redshift sources, which was hinted at in early interferometric studies (Chapman et al. 2004; Sakamoto et al. 2008; Ivison et al. 2012). This has now been clearly demonstrated by ALMA: while the typical extent of the starburst seen in local ULIRGs is of the order of a few 100's pc to $\sim 1 \mathrm{kpc}$, the rest-frame far-infrared emission in high-redshift SMGs arises from a region with an effective FWHM of $\sim 2-3 \mathrm{kpc}$ (e.g. Simpson et al. 2015b; Hodge et al. 2016). There are also hints that the dust continuum morphologies of some high-redshift SMGs show features which are not found in the typically more complex local counterparts. Thus recent high-resolution (0.03-0.3 arcsec) studies with ALMA have found that the dust continuum emission in SMGs has a disc-like brightness profile (Simpson et al. 2015a; Hodge et al. 2016; Ikarashi et al. 2017; Gullberg et al. 2018). While a study of six SMGs at $z \simeq 2.5$ by Hodge et al. (2019) at 0. '07 resolution $(\sim 0.5 \mathrm{kpc})$ has shown spatially resolved $870 \mu \mathrm{m}$ dust continuum morphologies with 'clump-like' structures bracketing elongated nuclear emission, reminiscent of bars and rings (Kormendy 2013). The sizes of the 'bars' and 'rings' are in the ratio of $1.9 \pm 0.3$, consistent with that expected for Lindblad resonances. If these are indeed bars and rings then analytic theory and numerical simulations (e.g. Binney \& Tremaine 1987; Lynden-Bell 1996; Athanassoula 2003) have shown that the ring is formed by gas outside the point of co-rotation being driven outward, by angular momentum transfer, collect into a ring near the outer Lindblad resonance. At radii inside the point of corotation, however, the gas falls inwards to the centre creating the bar. A bar is a means to drive gas from the outer part of the galaxy towards the centre, as the incoming gas is robbed of its energy, due to shocks. Gas can be funnelled inwards by the bar over an extended period, so maintaining the star formation in the central region. Simulations have suggested, however, that a bar can also cause quenching of star formation in the central region by sweeping up the gas within the co-rotational radius within a few rotations, which is then consumed in a vigorous burst of star formation (Gavazzi et al. 2015).

Hence, while SMGs and ULIRGs share some similar physical characteristics, the difference in the extent of their star formation, and current view of the dust continuum morphologies leaves open the possibility that the star-formation activity in the two populations are not driven by the same processes. Indeed, alternative theories have been proposed for how cold gas fuels the star formation in high-redshift starburst galaxies and how the star formation is triggered, through accretion from the cosmic web (Bournaud \& Elmegreen 2009; Dekel, Sari \& Ceverino 2009). In this scenario these galaxies rapidly accrete gas from the cosmic web and disc instabilities cause clumps to migrate to the nucleus where they form a bulge. These theories predict star-formation rates similar to the gas accretion rate of $\sim 100 \mathrm{M}_{\odot} \mathrm{yr}^{-1}$ and a resulting morphology of a gas disc twice the size of the nuclear star-forming bulge (Dekel et al. 2009).

To improve our understanding of the dust continuum structures of strongly star-forming galaxies at high redshift and so throw light on their possible formation and triggering mechanisms, we have analysed the morphologies of a much larger sample of SMGs to those studied to date. In this paper we present the result of this high-resolution (0.18) continuum study of the $870 \mu \mathrm{m}$ morphology of 153 SMGs from the AS2UDS ALMA survey of sub-millimetre sources in the SCUBA-2 Cosmology Legacy Survey UDS field (Stach et al. 2019). This large sample of uniformly selected SMGs, with integrated continuum signal-to-noise ratios of $\geq 8$, provides a statistically robust constraint on the sizes and, for the first time, the shapes of this high-redshift population. By selecting only the highest resolution observations and applying a conservative signal-to-noise cut, we seek to go beyond measuring crude sizes for the SMGs and instead derive constraints on their profiles and axial ratios for large statistical samples. These can then be used to investigate the physical nature of the dust continuum emission in these systems. Our observations resolve the $870 \mu \mathrm{m}$ dust emission in these sources and so provide reliable measures of the shape and profile parameters, such as the effective radius $\left(R_{\text {eff }}\right)$, axial ratio distribution of the population and typical Sérsic indices.

An outline of the structure of this paper is as follows: in Section 2 we present the observations used for in our analysis. We analyse these and describe our basic results in Section 3. We then interpret and discuss these in Section 4, before giving our conclusions in Section 5. We adopt a standard concordance, flat $\Lambda$ cold dark matter cosmology of $H_{0}=71 \mathrm{~km} \mathrm{~s}^{-1} \mathrm{Mpc}^{-1}, \Omega_{\Lambda}=0.73$, and $\Omega_{\mathrm{M}}=0.27$ (Spergel et al. 2007). 


\section{SAMPLE SELECTION}

Our sample is drawn from an ALMA follow-up study, called AS2UDS (Stach et al. 2019), of the sub-millimetre sources discovered in the SCUBA-2 Cosmology Legacy Survey map of the Ultra Deep Survey field (S2CLS Geach et al. 2017). Details of the AS2UDS observations, data reduction, and catalogue are given in Stach et al. (2019), although we briefly summarize these here. Using ALMA in Cycles 1, 3, 4, and 5 we targeted a complete sample of 716 single-dish SCUBA-2 $850 \mu \mathrm{m}$ sources with observed flux densities of $S_{850}>3.6 \mathrm{mJy}$ (corresponding to $>4 \sigma$ detection significance in the SCUBA-2 map). For all the observations, the central frequency of the receivers was tuned to $344 \mathrm{GHz}$ and the FWHM of the ALMA primary beam was 17.3 arcsec (encompassing the FWHM of the SCUBA-2 beam of 14.7 arcsec).

To reduce the data, we used the Common Astronomy Software Application (CASA, McMullin et al. 2007) version 4.5.3 using the standard ALMA calibration scripts. The data were imaged using the CLEAN, algorithm in CASA with natural weighting (ROBUST = 2). We CLEANED the images to the $1.5 \sigma$ level. Due to configuration differences during these cycles, the FWHM of the naturally weighted synthesized beam varies from 0.18 to 0.35 arcsec (with a small number of repeat observations obtained at 0.7 arcsec in Cycle 5 to test if flux was being resolved out of the higher resolution maps, Stach et al. 2019). Hence to construct the catalogue, all of the maps were tapered to 0.5 arcsec FWHM. The noise in these tapered maps varies between 0.09 and $0.34 \mathrm{mJy}_{\text {beam }}{ }^{-1}$ (see Stach et al. 2019 for more information about the AS2UDS data reduction).

The final AS2UDS catalogue contains 706 SMGs that are detected at $>4.3 \sigma$ ( 2 percent false-positive rate) with a median flux density of $S_{870} \sim 3.7 \mathrm{mJy}$ (Stach et al. 2019). We note that in the tapered maps, on average we recover the majority of the singledish flux for sources with $S_{870} \gtrsim 3.5 \mathrm{mJy}$ (Stach et al. 2019).

For this morphological study of the dust emission in SMGs, we concentrate on the Cycle 3 observations where a subset of 507 SMGs from the AS2UDS survey were detected in maps at a native resolution of 0.18 arcsec FWHM. For relatively high-resolution observations similar to these, Simpson et al. (2015b) showed that for a signal-to-noise ratio of $\mathrm{S} / \mathrm{N}>8$, the uncertainties on the resulting size measurements of sources are $\lesssim 35$ percent (Simpson et al. 2015b). We therefore select all 153 SMGs which were observed in Cycle 3 (0.18 arcsec FWHM) and are detected with $\mathrm{S} / \mathrm{N}>8$ in the 0.5 arcsec tapered maps, and these form the sample for the remainder of our analysis. This selection should ensure we can measure robust sizes and shapes for these sources and that we are sensitive to a broad range in source sizes. This sample of $153 \mathrm{SMGs}$ has a median flux density of $S_{870}=5.6 \pm 0.2 \mathrm{mJy}$, roughly $\sim 50$ per cent brighter than the full sample, with a range in flux density of $2.9-11.9 \mathrm{mJy}$, spanning the bulk of the SMG population which has been studied with ALMA. This means that though the exposure time of $40 \mathrm{~s}$ per source is short, we reach similar $\mathrm{S} / \mathrm{N}$ levels as studies of fainter SMGs but with longer exposure times (e.g. Tadaki et al. 2017).

Our ALMA survey was carried out in the $\sim 1 \mathrm{deg}^{2}$ UDS field, part of which was observed with Hubble Space Telescope (HST) by the Cosmic Assembly Near-infrared Deep Extragalactic Legacy Survey (CANDELS; Grogin et al. 2011). This provides $F 606 W-, F 814 W$ , $F 125 W$-, and $F 160 W$-band observations for 47 of the AS2UDS SMGs that lie in the central region of the UDS. We compare the optical/near-infrared and $870 \mu \mathrm{m}$ dust continuum morphologies of some of these galaxies in Fig. 1. This shows HST IJH-band colour thumbnails of eight ALMA SMGs, overlaid with the dust continuum emission from ALMA. At the median redshift of our sample $(z \sim 3)$, the observed $I J H$ bands samples the rest-frame mid-UV to $B$-band, and as Fig. 1 shows, the rest-frame UV/optical morphologies display a range of structures on arcsecond-scales from disc-like to apparently multicomponent mergers, point sources, and SMGs that are undetectable in even the reddest HST filters. In contrast, on average, the $870 \mu \mathrm{m}$ continuum appears much more compact than the rest-frame UV/optical emission, although generally the emission in the two wavebands is centred in the same position.

\subsection{Multiwavelength data sets and physical properties of the sample}

Before we assess the dust continuum sizes of our SMG sample, for context we review the physical properties of our high-resolution subsample from AS2UDS and place them in context of the parent population of 706 ALMA SMGs in this field. In particular, Dudzevičiūte et al. (2019) use the extensive multiwavelength imaging of the UDS to estimate the photometric redshifts and physical properties of the complete sample, including inferring their stellar masses, starformation rates, and dust masses. To achieve this, Dudzevičiūtè et al. (2019) exploit 22-band photometry (or limits) for each SMG, ${ }^{1}$ building on the UDS DR11 $K$-band selected catalogue of Almaini et al. (in preparation), and fit the spectral energy distribution, including deriving the photometric redshift estimates and uncertainties, using the high-redshift version of MAGPHYS (da Cunha et al. 2015; Battisti et al. 2019).

From the analysis of the multiwavelength SEDs, Dudzevičiūte et al. (2019) determine that the median redshift of the full sample of 706 ALMA SMGs is $z=2.61 \pm 0.08$, with a quartile range of 1.8 3.4. The median star-formation rate determined for the full parent sample is $235 \mathrm{M} \odot \mathrm{yr}^{-1}$, the median dust mass is $6.7 \times 10^{8} \mathrm{M}_{\odot}$ and the stellar mass is $(1.3 \pm 0.1) \times 10^{11} \mathrm{M}_{\odot}$ (Dudzevičiūtè et al. 2019). The corresponding values for the sub-sample of 153 SMGs in our high-resolution sample are $z=2.9 \pm 0.1$, with a quartile range 2.5-3.5, a median star-formation rate of $380 \mathrm{M} \odot \mathrm{yr}^{-1}$, a dust mass of $1.1 \times 10^{9} \mathrm{M}_{\odot}$ and stellar mass of $(1.3 \pm 0.1) \times$ $10^{11} \mathrm{M}_{\odot}$. As expected, our high-S/N SMG sample, which are roughly 50 per cent brighter in $S_{870}$ than the full sample, also exhibit correspondingly higher dust masses and star-formation rates and due to the correlation between observed $S_{870}$ and redshift reported by Stach et al. (2019) means that lie at somewhat higher redshifts than the full sample. We will return to a discussion of the trends of dust continuum structure with flux and star-formation rate in Section 4.

\section{ANALYSIS AND RESULTS}

In this section, we first assess the spatial extent of the dust continuum emission in our high-resolution observations of SMGs through measurement in both the $u v$-amplitude plane, and fitting models to the image plane maps. We then derive the Sérsic profiles and axial ratios for the continuum emission.

\footnotetext{
${ }^{1}$ The multiwavelength imaging includes photometry from deep optical UBVRi $z^{\prime}$ imaging from Subaru and CFHT, near-infrared from UKIRT $(J H K)$ and VISTA $(Y)$, Spitzer IRAC 3.6-8.0 $\mu \mathrm{m}$ / MIPS $24 \mu \mathrm{m}$ mid-infrared imaging, deblended far-infrared photometry from Herschel PACS (100 and $160 \mu \mathrm{m})$ and SPIRE $(250,350$, and $500 \mu \mathrm{m})$, ALMA $870 \mu \mathrm{m}$ and JVLA $1.4 \mathrm{GHz}$.
} 

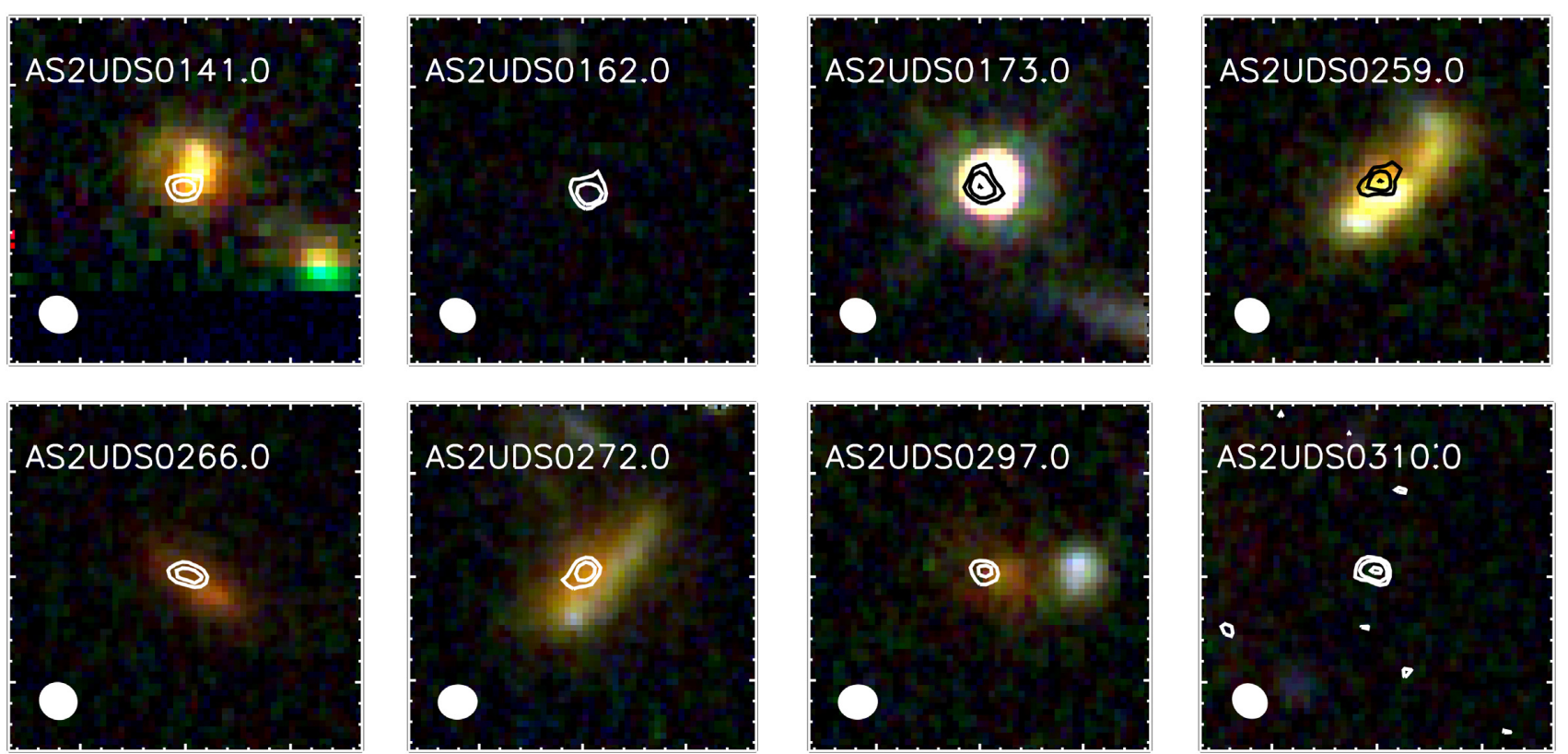

Figure 1. HST images $(I J H)$ of eight examples from the 153 SMGs in our survey, overlaid with $870 \mu \mathrm{m}$ dust continuum contours at $3 \sigma$, $5 \sigma$, and $9 \sigma$, with a median RMS level of $\sim 23 \mu \mathrm{Jy}$ The $870 \mu \mathrm{m}$ dust continuum emission in these eight SMGs show some similarities to their stellar emission, in terms of centroid and broad alignment, although the $870 \mu \mathrm{m}$ continuum emission is much more compact than the stellar emission. Each thumbnail is $3.4 \times 3.4$ arcsec, corresponding to $\sim 26 \mathrm{kpc}$ at the median redshift of the sample of $z \sim 3$ and the beam size is shown in the lower left corner.

\subsection{Sizes measurements from the visibility plane}

The spatial extent of the $870 \mu \mathrm{m}$ continuum emission of our SMGs can be derived by measuring the amplitude as a function of $u v$ distance. We apply this approach to each SMG by first aligning the phase centre of our visibilities with the source positions from Stach et al. (2019) using the CASA task FIXVIS and then radially averaging the amplitudes in $75 \mathrm{k} \lambda$ bins (the choice of $75 \mathrm{k} \lambda$ bins is arbitrary, although this binning minimizes the scatter). In Fig. 2 we show the real part of the amplitude as a function of $u v$-distance for the $u v$-range out to $1200 \mathrm{k} \lambda$ for the same eight galaxies shown in Fig. 1. The error bars on the amplitudes are given by the error on the mean in each bin. In this figure, we also include the total flux measurements from the 0.5 resolution $u v$-tapered maps.

Fig. 2 shows that in all cases, the amplitude declines as a function of increasing $u v$-distance. This is a clear indication that the emission from the source is resolved in these observations. Fitting Gaussian light profiles we derive a median FWHM size of $0.25 \pm 0.03$ arcsec.

A subset of our sample were observed at $1.1 \mathrm{~mm}$ with $\sim 0.7$ arcsec resolution using ALMA by Ikarashi et al. (2017) who studied a sample of millimetre sources selected from the 1.1-mm AzTEC map of the UDS field. Unsurprisingly the sources in this bright 1.1-mm sample overlap with brighter $870 \mu \mathrm{m}$ sources in the S2CLS map and as a result 65 of the 69 sources in Ikarashi et al. (2017) are also included in AS2UDS, of which 30 are in our high-resolution 0.18 arcsec sub-sample. We compare the ratio of the estimated sizes from $u v$-fits to the $870 \mu \mathrm{m}$ and the lower resolution (but $\mathrm{S} / \mathrm{N}>10$ ) $1.1 \mathrm{~mm}$ observations for these 30 sources and derive a median ratio of FWHM from Gaussian fits of $0.95 \pm 0.05$. This provides strong independent confirmation of the reliability of our derived sizes using a completely independent observations, reduction and analysis method.

One noteworthy feature of Fig. 2 is that it is clear that the amplitude does not converge to zero at large $u v$-distances in many cases. Indeed, in 119 SMGs (out of 153) the amplitude is non-zero (at $>3 \sigma$ ) at $1200 \mathrm{k} \lambda$. Naively this would suggest that a large fraction ( $\sim 80$ per cent) of sources contain an unresolved component (compared to a Gaussian model) comprising on average $13 \pm 1$ per cent (or typically $S_{870}=0.63 \pm 0.05 \mathrm{mJy}$ ) of the emission.

Large $u v$-distances correspond to small physical scales, and so this 'compact' emission seen in Fig. 2 must arise on $\lesssim 0.18$ arcsec scales. One option is that the our observed $u v$-amplitude profile comprises a luminous, extended (Gaussian-like) starburst with a $\sim 13$ per cent contribution from a central point-source (Tadaki et al. 2017). However, it is also possible that the 'compact' emission instead arises from a light profile that is more centrally concentrated than a Gaussian (which is generally a poor description of the light or mass profiles of resolved galaxies). To investigate how different light profiles should appear in the $u v$-amplitude plane, in Fig. 3 we show the simulated $u v$-amplitude-distance as a function of Sérsic index (n) with $n=0.5,1$, and 2 and the median composite profile of the 153 SMGs in our sample. These profiles were created using the CASA simulation tool with the same configuration as our observations (and hence the same synthesized beam FWHM). For brightness profiles with Sérsic index $n>0.5$ the $u v$-amplitude profile does not converge to zero by a $u v$-distance of $\sim 1200 \mathrm{k} \lambda$ - a consequence of the steep central light profile which gives rise to apparently compact emission. Hence, fitting a Gaussian model to a marginally resolved source with an intrinsic Sérsic $n=1$ profile you would conclude that a second compact emission component was implied by the non-zero amplitude at large $u v$-distance. To illustrate the difference in Fig. 2 we overlay the best-fitting models with $n=0.5$ and $n=1$ to the eight galaxies shown. The $\chi^{2}$ distributions of the individual $n=0.5$ and $n=1$ fits show a moderate preference $(2.5 \sigma)$ towards the $n=1$ fits. This is supported by the composite profile, which is best fitted with a $n=1$ profile. The $n=1$ fits results in $\chi^{2} \sim 1.5$, compared to $\chi^{2} \sim$ 2.5 for a $n=0.5$ fit. As we show in Section 3.2 fitting a Sérsic model to each of the SMGs in our sample suggests a median Sérsic profile of $n=1.00 \pm 0.12$. This indicates that the majority ( 77 per cent) of 

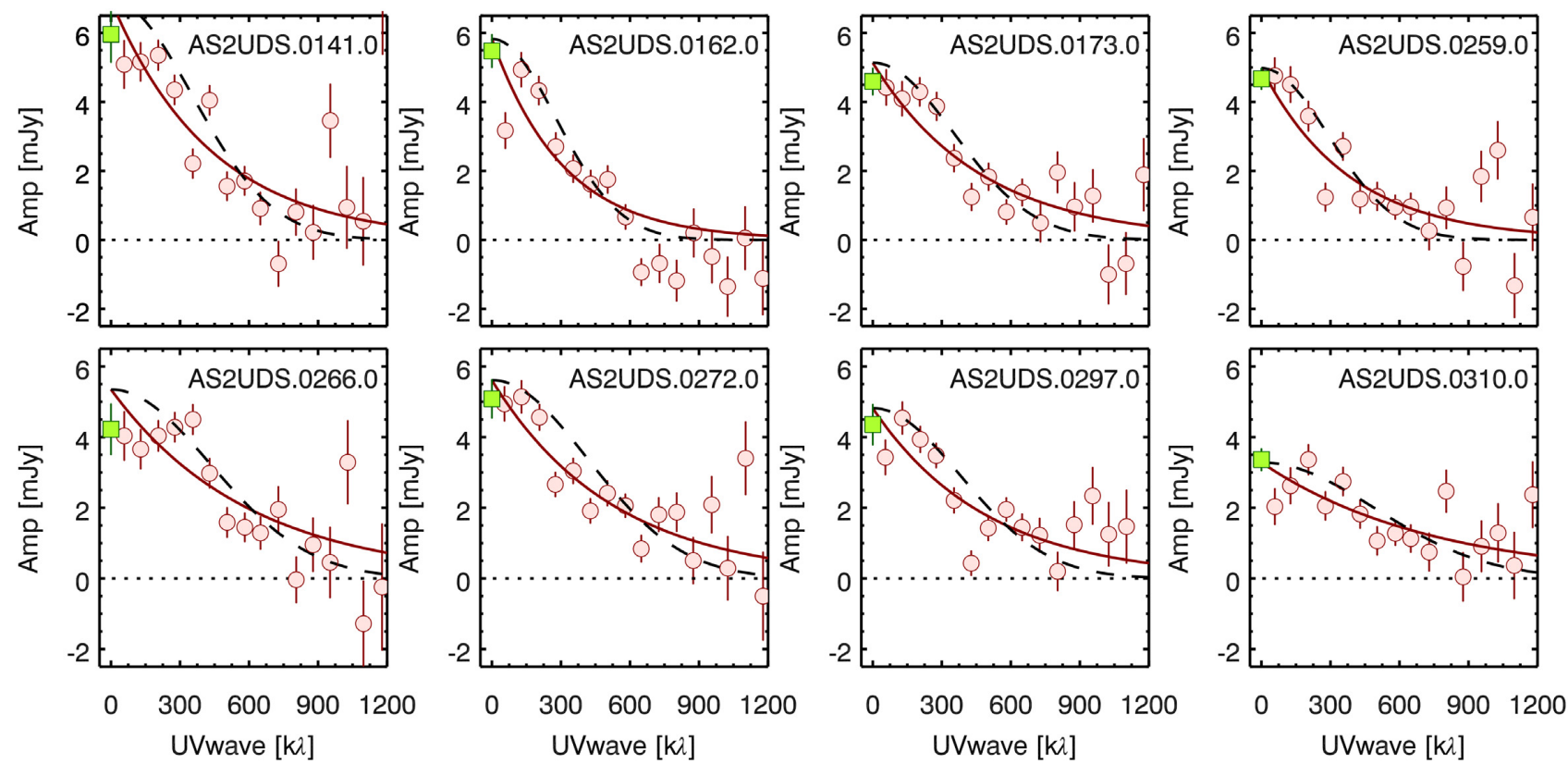

Figure 2. Visibility amplitudes (real part) as a function of the $u v$-distance for the eight SMGs shown in Fig. 1. The amplitudes are extracted by radially averaging the visibilities in $75 \mathrm{k} \lambda$ bins over the full frequency range and the total flux densities recovered in the maps $u v$-tapered to 0.5 arcsec resolution are plotted as a square. We overlay half-Gaussian fits to the continuum emission as a dashed lines, and a Sérsic fit with $n=1$ by the solid curve. The $870 \mu \mathrm{m}$ dust continuum of the SMGs are all resolved in our observations. We note that the Gaussian fits frequently show an apparent compact or unresolved component, indicated by a non-zero flux at large $u v$-distances. However, this is an artefact of the fact that the profiles are poorly described by a Gaussian, while an $n=1$ Sérsic better reproduces both the compact and extended emission. The $870 \mu \mathrm{m}$ dust continuum sizes of the sources are listed in Table A1.

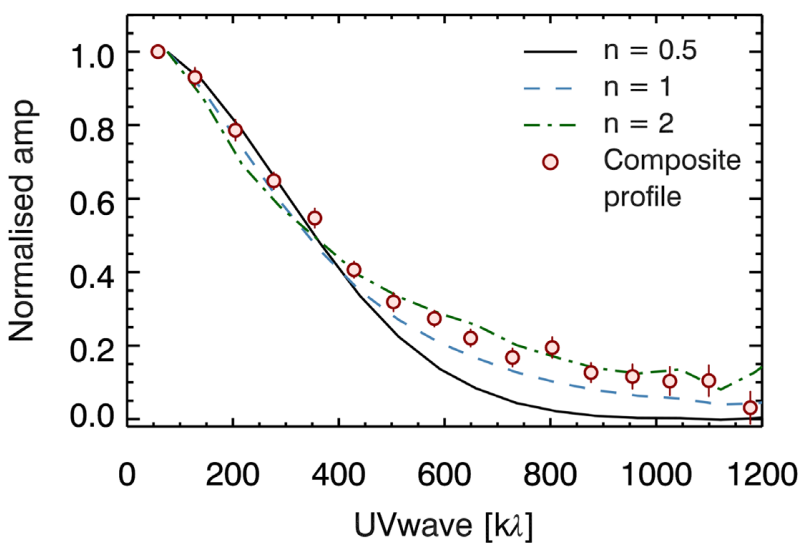

Figure 3. Visibility amplitudes as a function of the $u v$-distance for three model profiles with $n=0.5,1$, and 2 and radius of 0.15 arcsec, observed with the same array configuration set-up as our observations, compared to the median composite profile of our 153 SMGs. This illustrate that for a source with an intrinsic Gaussian profile $(n=0.5)$ the amplitude quickly converges to zero, while for higher $n$ the amplitude at the largest $u v$ distances remains non-zero out to at least $1000 \mathrm{k} \lambda$, mimicking the signal of a point-source component. This implies that for marginally resolved galaxies with profiles steeper than $n>0.5$ the inner part of the galaxy will appear unresolved, incorrectly suggesting the presence of a point-source component.

the SMGs in our sample have $870 \mu \mathrm{m}$ continuum profiles that are consistent with Sérsic with $n \sim 1$ (rather than Gaussian, $n \sim 0.5$ ) light profiles. We therefore conclude that Sérsic models provide an appropriate description of the $870 \mu \mathrm{m}$ brightness profiles of the SMGs in our sample. Hence to measure the spatial extent of the dust continuum emission, we adopt an $n=1$ Sérsic and allow the effective radius as a free parameter and fit the $u v$-amplitude profile for each SMG. We derive a median effective radius for the 153 SMGs in our high-resolution/high-S/N ratio sample of $0.10 \pm 0.04$ arcsec.

The typical effective radius we measure is comparable to estimates from previous studies. For example, Hodge et al. (2016) measure the spatial extent of the $870 \mu \mathrm{m}$ dust continuum in $16 \mathrm{SMGs}$ from ALMA observations with $\sim 0$ '. 16 resolution and a sample with flux density range of $S_{870}=3.4-9.0 \mathrm{mJy}$. They derive a median effective radius of $0.15 \pm 0.03$ arcsec, which is similar to our sample. Simpson et al. (2015a) also measure the $870 \mu \mathrm{m}$ sizes of 30 from our ALMA Cycle 1 observations of the brightest SMGs in the AS2UDS pilot (there is no overlap in sources to the 153 SMGs analysed here). Using that $\sim 0.3$ arcsec resolution data, Simpson et al. (2015a) derive a median effective radius of $0.13 \pm 0.02$ arcsec. While, as noted earlier, Ikarashi et al. (2017) analysed ALMA 1.1mm observations of 65 SMGs from AS2UDS and derive an effective radius of $0.13 \pm 0.06$ arcsec using their lower resolution, 0.7 arcsec data. Thus it appears that the extent of the dust continuum emission we measure for our sample is comparable to that estimated from earlier smaller scale studies.

\subsection{Sizes and shape measurements from the image plane}

The azimuthally averaged amplitude measured from the visibility plane is well suited for deriving a characteristic radius for the emission from a galaxy, but provides a circularized average. Information about the Sérsic index and the axial ratio $(b / a)$ of the emission can also be derived, in a computationally more tractable manner, from the image plane.

To measure the sizes from the image-plane maps of each SMG we fit a two-dimensional Sérsic surface brightness profile, allowing the effective radius, axial ratio and Sérsic index to vary. We account for 
the beam by convolving each intrinsic model with the synthesized beam with a semimajor and semiminor axes and position angle given by the beam parameters for each map. The fit returns measurements of the peak flux, the central position, the semimajor axes, the axial ratio (i.e. the ratio of the minor to major axes, $b / a$ ), the position angle, and for the case of free Sérsic fit, the Sérsic index $(n)$ as well as uncertainties on all these parameters.

Before we present the results of the fitting, we first test the reliability of the deconvolved measurements, and calibrate their uncertainties. To do this, we generate a set of 1000 simulated galaxies using CASA which have a flux distribution similar to our sample. These simulated galaxies have semimajor axis between 0.11 and 0.24 arcsec, and random inclination angles and Sérsic index. We use CASA to simulate the observations of these galaxies with the same exposure time as our data, and hence these simulated maps have similar noise properties as our sample. We then fit these simulated galaxies with our code and return their best-fitting parameters. On average we recover the effective radii to within 20 percent and the axial ratios are recovered within 12 percent of the value of the input parameters. The Sérsic index is the most difficult parameter to fit and recover at the signal to noise of our typical sources, with a typical error of 22 per cent. To investigate the potential bias due to noise when measuring the shapes of round sources (which may cause an observed decline in the apparent numbers of round sources), we also test the code on Gaussian profiles with axial ratio $b / a=1$ and find that the procedure with free fit returns the Sérsic index within 25 percent and the axial ratio within 14 per cent. The axial ratio distribution peaks for $b / a=$ 0.86 and has a standard deviation of 0.12 for a sample matched in signal-to-noise to our observations. We therefore conclude that the strong peak in axial ratio at $b / a \sim 0.65$ is our observed distribution if not a result of this bias.

The measurement of the Sérsic can also be influenced by data sampling (e.g. Robotham et al. 2017), and so next we investigate the influence of the reconstructed map pixel sampling by testing the same procedure with different pixel scales. We create 1000 model maps of SMGs at the same pixel scale as our data $\left(0.03 \operatorname{arcsec}_{\text {pixel }}{ }^{-1}\right)$, and also at three times smaller sampling $\left(0.01\right.$ arcsec pixel $\left.^{-1}\right)$. We simulate observations of these maps with CASA and then fit these maps and infer their properties. This test shows that finer oversampling of the synthesized beam does not return more accurate or precise Sérsic indices $\Delta n / n=0.34 \pm 0.05$. For low signal-to-noise profiles with input Sérsic indices $n \gtrsim 1.25$ the fitting-procedure on the oversampled maps return systematically lower values of $n$, but with increasingly larger uncertainties.

To derive measurements of the effective radii of the dust continuum in our SMGs, we now perform two sets of fits. First, we perform a fit with Sérsic $n$ as a free parameter. In these fits, we derive a median $n=1.00 \pm 0.12$ and $R_{\text {eff }}=0.11 \pm 0.01$ arcsec. Since the Sérsic index is the least certain parameter, we then fix the Sérsic index to $n=1.0$ and refit each SMG. For this $n=$ 1.0 fit (see Fig. A1), the median effective radius for the sample is $R_{\text {eff }}=0.10 \pm 0.04 \operatorname{arcsec}$ (see Fig. 4).

In Fig. 4 we compare the effective radius for the SMGs derived from the $u v$-fitting with that derived in the image plane. We first compare the effective radii derived from the fixed $n=1$ Sérsic fit in both cases, deriving a median ratio of the image-plane to $u v$-plane of $R_{\text {eff }}(u v) / R_{\text {eff }}(\mathrm{im})=1.10 \pm 0.01$. Although the two measurements are correlated, it is also clear from Fig. 4 that when fitting a Sérsic model in the image plane, for larger effective radii, the $u v$-derived effective radius is $\sim 30$ per cent larger than that derived in the image-plane, but with no strong trend with Sérsic index.
In Fig. 4 we compare the effective radii of the dust continuum in the image-plane for the free and fixed Sérsic models. The median ratio in effective radii of $R_{\text {eff }}$ (free) $/ R_{\text {eff }}(n=1)=1.07 \pm 0.01$. The scatter in this relation can be attributed to those SMGs with profile with a higher Sérsic (as indicated by the colour scaling of the points, which show that for higher Sérsic index, the free fit returns larger sizes). We also show the Sérsic index distribution derived from the free fit, which has a median of $n=1.00 \pm 0.12$. This shows that $\sim 70$ percent of the SMGs in our sample have Sérsic indices $n=$ $0.7-2$ (as also suggested by the $u v$-amplitude profiles).

Using the image-plane fits, we extract the distribution of axial ratios from the best-fitting models from both the free and fixed Sérsic model fits, and show these in Fig. 4. Both distributions are strongly peaked, with a median of $b / a=0.63 \pm 0.02$ for the fixed $n$ fit and $b / a=0.64 \pm 0.02$ for the free $n$ fits, and both distributions have the same standard deviations of $\sigma_{\mathrm{b} / \mathrm{a}}=0.19$. We confirm that there is no correlation between measured $b / a$ and effective radius.

Hodge et al. (2016) present axial ratios for their 16 ALESS SMGs observed at $870 \mu \mathrm{m}$ with 0 '.16 resolution, which span a narrow range in $b / a=0.3-0.7$ and have a median of $b / a=0.55 \pm 0.06$ and $\sigma_{b / a}=0.13$. Their distribution is thus consistent with that from our larger sample, and both display a relative lack of 'round' sources (compared to the naive expectation of randomly orientated circular, thin discs, Ryden 2004). We will return to a discussion of this distribution in Section 4.

In summary, both the $870 \mu \mathrm{m}$ dust continuum in the $u v$-amplitude profiles and image-plane maps suggest that the majority of the SMGs in our sample are best fit by $n \sim 1$ Sérsic models. Fitting Sérsic models to the $u v$ and image plane returns consistent results, and suggests a median effective radii of $0.10 \pm 0.04$ arcsec $(\sim 0.8 \pm 0.3 \mathrm{kpc}$ at the median redshift of our sample). Since the Sérsic fit in the image-plane maps also allows us to easily investigate the shape parameters for the SMGs, for the remaining analysis we will use this method, but will adopt the minimum uncertainties on any measurement of effective radius from the scatter determine from the correlation(s) in Fig. 4.

\subsection{Comparison of the dust and rest-frame optical emission}

In Fig. 1 we show the HST images of eight example SMGs in our sample which are also observed as part of the CANDELS survey (Grogin et al. 2011). It is clear from this figure that the rest-frame optical emission is much more extended than the $870 \mu \mathrm{m}$ emission (see also Simpson et al. 2015a; Chen et al. 2015; Lang et al. 2019). To quantify this further, we perform a Sérsic fit to the $K$-band images of all of the SMGs in our sample using GALAPAGOS (Almaini et al. 2017, Maltby et al. in prep) which takes the PSF into account, and we compare the effective radii measured in the UDS $K$-band compared to that measured from $870 \mu \mathrm{m}$ in Fig. 4. This shows that for galaxies with $K$-band axial ratio of $b / a=$ $0.75-1$ (i.e. close to circular) the $K$-band effective radii are a factor of $2.2 \pm 0.2$ larger than the effective radii measured at $870 \mu \mathrm{m}$, although with considerable scatter (especially for those with large apparent axial ratios). This implies that the stellar light distribution is typically twice that of dust (see also Lang et al. 2019). We will discuss the extended emission in the stars and dust further in Section 4. We note, however, that optical depth effects need to be considered, in cases of more detailed comparisons of sizes measured at different wavelengths, for example between short-wavelength dust emission, and [C II] or low or high-J CO emission. 

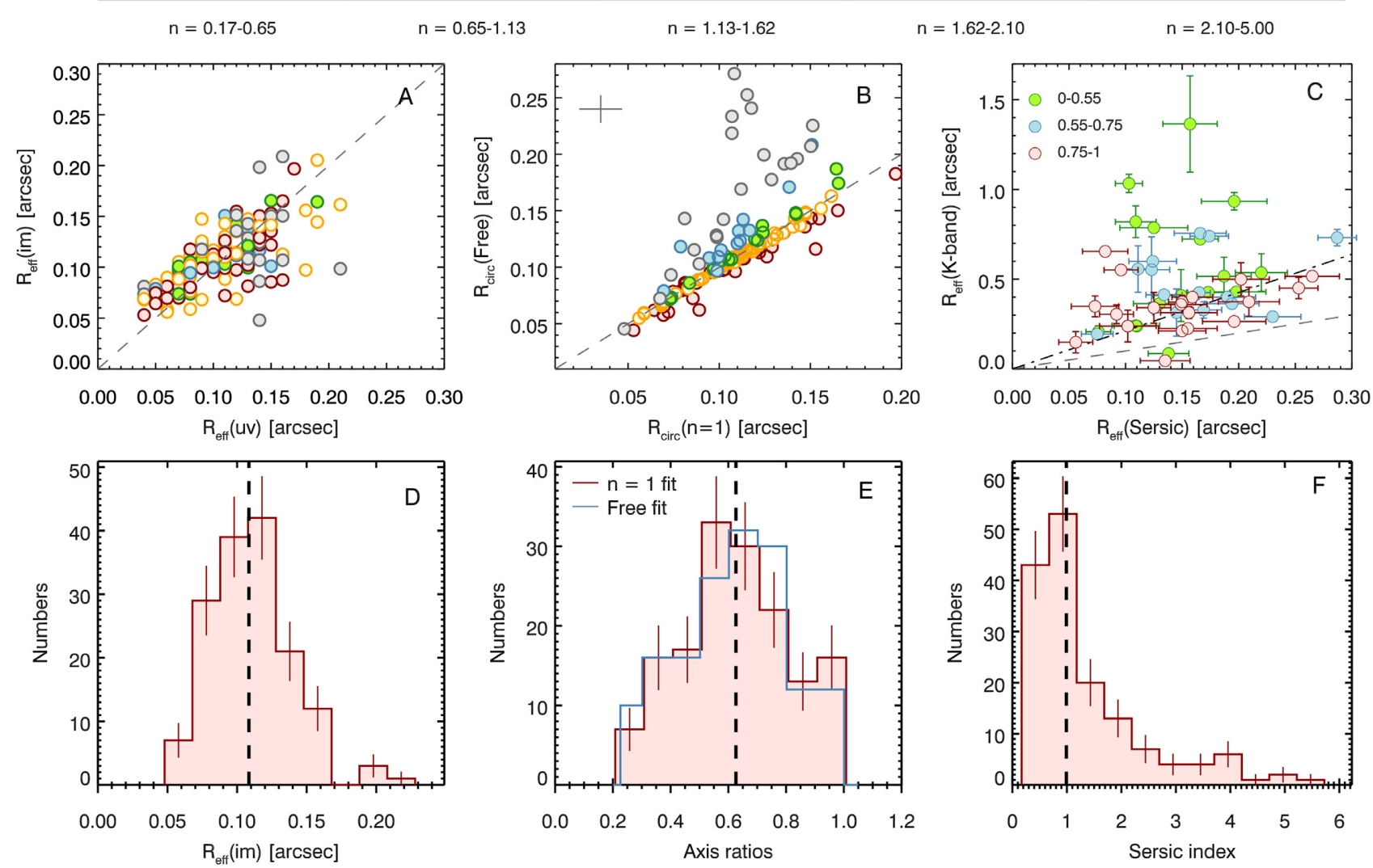

Figure 4. A: Effective radii $\left(R_{\text {eff }}\right)$ values measured in the visibility plane (with $\left.n=1\right)$ compared to the circularized $R_{\text {eff }}$ measured using Sérsic fits with fixed $n=1$ in the image plane. The $R_{\mathrm{eff}}$ measurements from the two methods show a one-to-one correlation, with a scatter partly due to the difference in profile. Profiles close to $n \sim 1$ show better agreement than profiles with $n \sim 2-5$. B: Comparison of the circularized $R_{\text {eff }}\left(R_{\text {circ }}\right)$ measurements from the $n=1$ and free Sérsic fits. For $n \lesssim 1.6$ the radii for the $n=1$ and free Sérsic fits agree within $1 \sigma$, while at $n \gtrsim 1.6$ the free Sérsic fit returns up to $\sim 2.5$ times higher $R_{\text {circ }}$ than the $n=1$ fit. $C$ : Effective radii measured in $K$-band imaging as a function of $R_{\text {eff }}$ we measure with the free Sérsic fit. The points are coloured according to the measured axial ratios in the $K$-band images. When the sources appear close to circular, axial ratios $0.75-1$, in the $K$-band image, the measurements show a rough correlation (indicated by the dash-dotted curve) with a relative size ratio $(K$ to $870 \mu \mathrm{m})$ of $2.2 \pm 0.2$. More elliptical $K$-band measurements show a larger scatter, which (by assuming disc morphologies) perhaps indicate that the $K$-band imaging is more affected by extinction when viewed edge-on or the geometry of the dust and stellar light is different and hence varies as function of orientation. We return to this point in Section 3.5 when we model the structure of the dust in these galaxies and show that the dust morphology does not appear to be disc like. $D$ : Distribution of circularized $R_{\mathrm{eff}}$ for the $n=1$ Sérsic fit, with a median $R_{\text {eff }}$ of $0{ }^{\prime} 10 \pm 00^{\prime \prime} 0.04$. E: The distribution of the axial ratios for the $n=1$ fit overlaid with the axial ratio from the free Sérsic fit. The $n=1$ fit distributions has a median of $\sim 0.64 \pm 0.24$, and the free fit distribution has a median of $0.63 \pm 0.24$. F: The Sérsic index distribution of the free fit has a median Sérsic index of $n=1.00 \pm 0.12$. The majority of the SMGs have $n<1.5$, however, the distribution shows a tail out to $n \sim 5$.

\subsection{Size and shape evolution}

Previous morphological studies (with sufficiently high signal-tonoise detections) of SMGs at sub-/millimetre wavelengths have been limited either by moderate resolutions (Simpson et al. 2015b; Ikarashi et al. 2017) or modest sized samples (Hodge et al. 2016; Gullberg et al. 2018; Hodge et al. 2019). Our sample of 153 SMGs detected at $\mathrm{S} / \mathrm{N}>8$ in 0 '. 18 resolution maps, allow for a statistical study of the morphology, sizes and axial ratios for a wide range in both $870 \mu \mathrm{m}$ flux density $\left(S_{870} \sim 2.7-11.5 \mathrm{mJy}\right)$ and redshift $(z \sim 1-6)$.

Our sample has a median redshift of $z=2.9 \pm 0.1$ and a quartile range in redshift of $2.5-3.5$, so the corresponding restframe wavelength $\left(\lambda_{\text {rest }} \geq 150-300 \mu \mathrm{m}\right)$ of the dust emission from the galaxies should be generally optically thin (e.g. Simpson et al. 2017). However, at the highest redshift, the dust emission may be optically thick (e.g. at $z \gg 3.5-4$, the rest-frame wavelength is $\lambda<200 \mu \mathrm{m}$ ), this would then produce a small bias such that galaxies whose orientation provides a larger apparent sky area (i.e. face-on for disc-like geometries) would have brighter $S_{870}$. There is a possible hint of this in Fig. 5 where we plot the axial ratios as a function of both $870 \mu \mathrm{m}$ flux density and redshift (Dudzevičiūtè et al. 2019), and find weak positive correlations.

In the plot of effective radius $\left(R_{\text {eff }}\right)$ versus $870 \mu$ m flux density, we identify a weak positive correlation. But this trend is marginal and so we conclude that the brighter SMGs are more luminous primarily due to their higher dust surface densities, rather than their larger sizes. The apparent trend suggests a doubling of dust mass surface density between SMGs with $S_{870} \sim 4-11$, which may imply a similar increase in gas density and a corresponding rise in midplane pressure in these systems, which are already thought to be extremely high (Swinbank et al. 2011, 2015).

More interestingly, we see a small variation of the effective radius of $\sim 10$ percent (corresponding to $\sim 2.5 \times 10^{8} \mathrm{M}_{\odot} \mathrm{kpc}^{2}$ ), by a weak decline with redshift (Dudzevičiūtè et al. 2019). We 

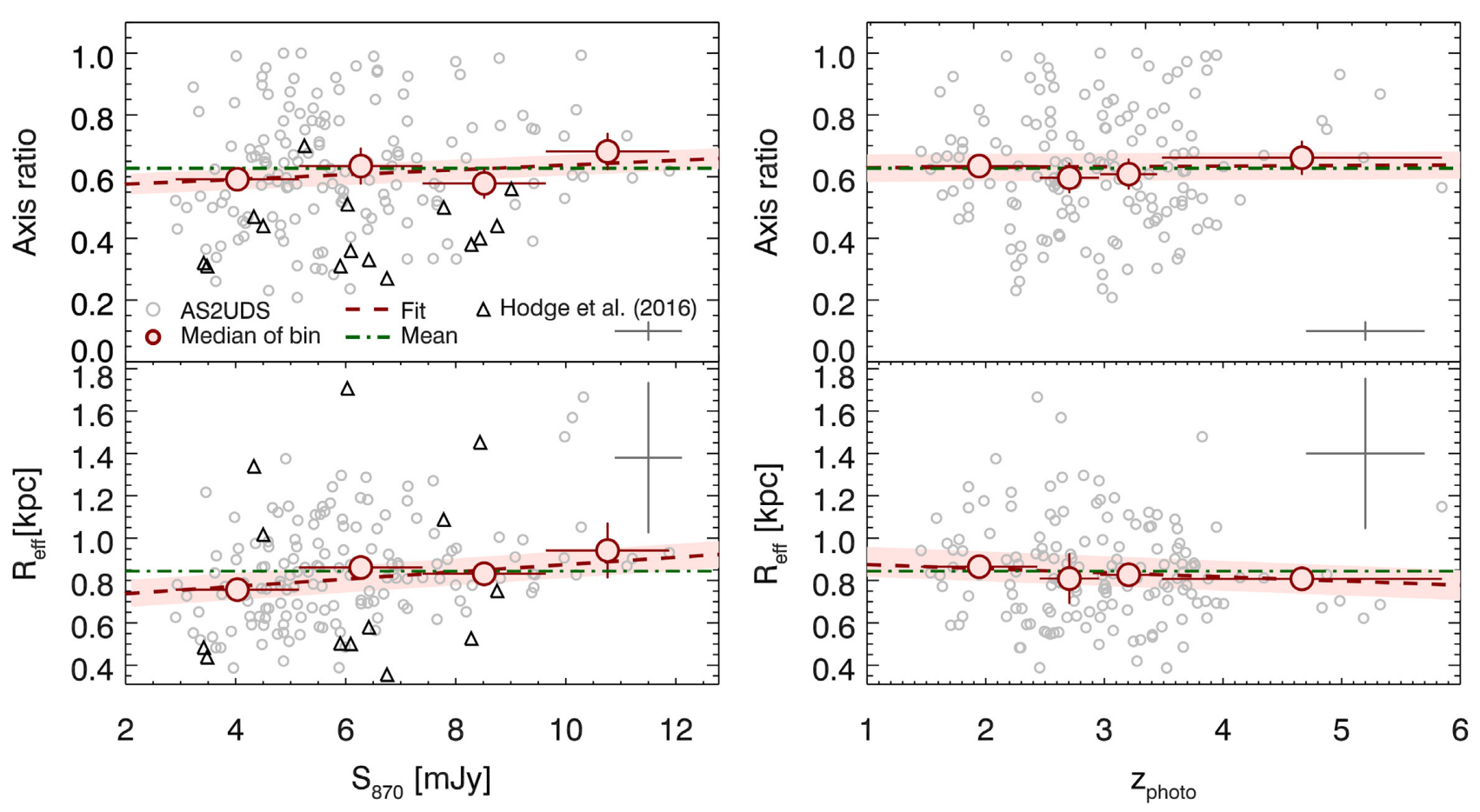

Figure 5. Left: Measured axial ratios (top) and $R_{\text {eff }}$ (bottom) from the $n=1$ fit as functions of the $870 \mu \mathrm{m}$ continuum flux density. The large points are the medians in bins of equal numbers of sources. We see weak positive correlations between both the axial ratio and $R_{\text {eff }}$ with $870 \mu \mathrm{m}$ continuum flux density (dashed curves). Right: Measured axial ratios (top) as function of the photometric redshift ( $z_{\text {photo }}$; Dudzevičiūte et al. 2019) shows a very weak positive correlation, while the $R_{\text {eff }}$ (bottom) decreases marginally as a function of the photometric redshift. These data are observed $870 \mu \mathrm{m}$ meaning that the rest-frame wavelengths vary across the redshift range $z \sim 1-6$, and hence the effective optical depth is expected to vary at the observed wavelength. The influence of these optical depth effects, as well as potential evolution in the structure and physical properties of the SMGs (e.g. dust mass and far-infrared luminosity) mean it is hard to draw strong conclusions from this plot. Nevertheless, we suggest that the lack of any strong trends in effective radius with redshift most likely indicates that there is no strong evolution in the size of the SMGs with redshift.

expect this behaviour to reflect both evolution in the physical size of the sources and also the influence of dust optical depth, dust temperature and source structure. For simple source geometries, the apparent sizes of sources are expected to decline with increasing $L_{\mathrm{FIR}} / M_{\mathrm{d}}$ (Falcón-Barroso \& Knapen 2013). Using the estimates of $L_{\mathrm{FIR}}$ and $M_{\mathrm{d}}$ for our sample from (Dudzevičiūte et al. 2019), we expect an increase in median $L_{\mathrm{FIR}} / M_{\mathrm{d}}$ of only $\sim 20$ percent in the sample across $z \sim 2-4$ (Falcón-Barroso \& Knapen 2013), which would correspond to a $\sim 20$ percent decline in apparent size at a fixed rest-frame wavelength. However, this will be countered by the effect of shifting to shorter (and hence optically thicker) rest-frame wavelengths as we observe higher redshift sources. Thus we expect the drop in observed $870 \mu \mathrm{m}$ effective radius with redshift to be less than $\leq 20$ per cent, which would be consistent with the weak decline seen in Fig. 5.

Overall, we conclude there are a number of potentially competing effects which could influence the variation in apparent size of the SMGs with redshift, but none of these effects is strong and hence the absence of significant evolution in the effective radius with redshift most likely indicates that the intrinsic physical sizes of the SMGs do not evolve strongly with redshift. This would be in contrast with studies in the UV and optical of a variety of galaxy populations which have reduction of a factor of several in typical size with redshift for both quiescent galaxies and star-forming galaxies across $z \sim 0-4$ (e.g. van der Wel et al. 2014; Shibuya, Ouchi \& Harikane 2015; Kubo et al. 2018).

\subsection{Modelling the axial ratio distribution}

Our results above, as well as recent studies (e.g. Simpson et al. 2015b; Hodge et al. 2016), have shown that the $870 \mu \mathrm{m}$ dust emission in SMGs follows an exponential surface brightness profile suggestive of a disc-like geometry. For a sample of circular exponential discs viewed at random viewing angles, the axial ratio distribution should be constant at high axial ratios, with a decline towards $b / a=0$, the strength of which depends upon the relative thickness of the disc. However, as shown in Fig. 4 for our SMGs, the apparent axial ratio distribution is highly peaked, with a large fraction of the sample $(62 \pm 4$ percent) having axial ratio in the range $b / a=0.5-0.8$, and proportionally fewer at $b / a<0.5$ and $b / a>0.8$, where the latter account for $15 \pm 8$ percent of the sample. This 'deficit' in the number of sources with near-circular shapes suggests that either the assumption of SMGs being circular discs with a uniform viewing angle distribution is incorrect, or that signal-to-noise effects cause us underestimate the numbers of high b/a sources.

We first confirm that the behaviour we see is not due to signalto-noise effects. To ensure that our profiles are robust, we set a lower limit for the signal to noise of $\mathrm{S} / \mathrm{N}>8$, since the fractional uncertainties on the measured sizes increases to over $\sim 35$ per cent at lower signal-to-noise ratios (Simpson et al. 2015b). The signalto-noise ratio of our sample ranges from 8 to 29 with a median of 12. Dividing the sample in half at $\mathrm{S} / \mathrm{N} \simeq 12$ yields two distributions which both show a 'deficit' of axial ratios at $>0.8$, suggesting that the signal-to-noise is not the cause of the observed 'deficit' of high 
axial ratio sources. Hence we now explore three geometrical models for the sources to attempt to reproduce the observed axial ratio distribution (including the influence of potential selection effects): optically thick discs, optically thin discs and a model with a triaxial geometry for the sources.

\subsubsection{Geometrical models}

To assess the possible influence of selection effects on the axial ratio distribution we compare our observed axial ratio distribution with those predicted for sources which are modelled as optically thick or thin circular discs. We generate a simulated sample of circular discs, where the apparent axial ratio is only dependent on the (random) viewing angle. We follow the example of Ryden (2004), where the apparent axial ratio $q$ is given by

$q=\left[\frac{A+C-\sqrt{(A-C)^{2}+B}}{A+C+\sqrt{(A-C)^{2}+B}}\right]^{1 / 2}$,

where $A, B$, and $C$ are given by

$A=\left[1-\epsilon(2-\epsilon) \sin ^{2} \phi\right] \cos ^{2} \theta+\gamma^{2} \sin ^{2} \theta$,

$B=4 \epsilon^{2}(2-\epsilon)^{2} \cos ^{2} \theta \sin ^{2} \phi \cos ^{2} \phi$,

$C=1-\epsilon(2-\epsilon) \cos ^{2} \phi$.

Here $\epsilon$ is the ellipticity of the source $\epsilon=1-b / a$ (where $a$ and $b$ are the intrinsic major and minor axes), and $\gamma$ is the ratio between the third axis $(c)$ and the major axis; $\gamma=c / a$. The two angles $\theta$ and $\phi$ are the two viewing angles. Only $\theta$ has an influence on the apparent axial ratio in the case of a circular disc, where $a$ and $b$ are equal.

The resulting distribution of apparent axial ratios for circular discs is therefore dependent on the distribution of the viewing angle $\theta$, the $c / a$ ratio, and the flux distribution. We assume a flux distribution similar to our sample and calculate the apparent axial ratios for the possible combinations of the different parameters, adopting a uniform distribution for the viewing angle and for $c / a$.

Model I. Optically thick disc - In the case of an optically thick disc, at an observed wavelength of $870 \mu \mathrm{m}$, the fraction of the emitted emission that is detected by the observer is given by the visible fraction of the surface area (i.e. the apparent axial ratio).

We attempt to fit the distribution of $b / a$ with equation (1) for this optically thick model, but in all cases the best fit is poor (see Fig. 6). The best-fitting optically thick disc models with a uniform viewing angle distribution has a Gaussian-shaped $c / a$ distribution, peaking for $c / a=0.06$ with standard deviation of 0.33 . However, the best-fitting is still poor, and a Kolmogorov-Smirnov (KS) test shows a negligible ( 0 per cent) chance for these two distributions to be drawn from the same parent sample. While it would be possible to bias the viewing angle distribution and find a better fit, such nonuniform viewing angle distributions are unphysical in the absence of an identifiable selection bias as a cause, and so we discard this option. $^{2}$

Model II: Optically thin disc - For the optically thin case the emission only depends on the assigned source brightness. As for the optically thick case, we are unable to find acceptable fit to the observations for the optical thin model with a uniform viewing angle distribution. The closest model for the optically thin case, with a uniform viewing angle distribution, has a rise in axial ratio

\footnotetext{
${ }^{2}$ We note that our sample is selected from low-resolution single-dish observations and so is not expected to suffer from surface brightness selection biases.
}

distribution beginning at lower axial ratios, and has a flatter and slower rise than in the optically thick case. The best-fitting model with a uniform viewing angle distribution has a $c / a$ distribution peaking at 0.09 , with a standard deviation of 0.28 . Again a KS test returns a negligible chance ( 0 per cent) of the two distributions being drawn from the same parent sample. The only way to match the 'deficit' at high axial ratio would then be to include a biased viewing angle distribution, which as noted earlier is not physically plausible.

Model III: Triaxial structure - The shape of our observed axial ratio distribution differs from those seen for late-type spiral galaxies (Ryden 2004), which lack the strong peak at $b / a \sim 0.6$ and the associated deficit at high axial ratios which we observe. We have quantitatively confirmed this above and conclude that neither of the circular disc models is able to adequately fit the data without invoking assumptions of unphysical viewing angle biases. Thus we now explore a triaxial model, where $a>b>c$. In this model we determine the best-fitting $b / a$ and $c / a$ axial ratios distribution to fit the observed axial ratio distribution for an optically thin case. For the triaxial case both viewing angles $(\theta$ and $\phi)$ have an influence on the apparent axial ratio $q$. We assume uniform angle distributions for both $\theta$ and $\phi$, and model the apparent axial ratio distribution for a range of model and width parameters for the $b / a$ and $c / a$ distributions. We assume that the $b / a$ and $c / a$ distributions follow Gaussian distributions given by

$f\left(q_{\text {int }}\right)=\exp \left(-\frac{\left(q_{\text {int }}-q_{0}\right)^{2}}{2 \sigma^{2}}\right)$,

where $q_{\text {int }}$ represents the intrinsic $b / a$ and $c / a$ axial ratios, $q_{0}$ is the mode value, and $\sigma$ is the width of the distribution. We calculate axial ratios for two different ranges of mode and width parameters for $b / a$ and $c / a$ (see Table 1).

As the distributions are expected to be continuous and smooth, we perform our search for the best-fitting model by running through the parameter space twice; first by using a large bin size to cover the full parameter range and select the combination of parameters that provide the closest match to the observed axial ratio distribution; this best-fitting parameter combination is then used as the centre of the second run which uses a finer search grid. The parameters for the $b / a$ and $c / a$ distributions that result in an apparent axial ratio distribution best fit to our observed distribution (and see Fig. 6) are given in Table 1 (see also Fig. 6). A KS test shows that there is a 40 per cent chance that the triaxial axial ratio distribution and our observed axial ratio distribution originate from the same parent sample. This is further supported by the Akaike Information Criterion (AIC), which takes into account the number of fitted parameters, and for the triaxial case is 10.8. The AIC values for the optical thick and thin cases with uniform viewing angle (discussed in Section 4.4) distributions are 33.8 and 15.2. The model resulting in the lowest AIC values yields the best-fitting model, which is therefore the triaxial model. We conclude that the 'deficit' in high axial ratios is most likely due to intrinsic triaxial morphologies, rather than the dust continuum emission of SMGs resembling randomly orientated circular disc galaxies.

\subsection{Stacked emission profiles}

Near-infrared HST imaging of ALMA SMGs shows that the restframe UV/optical emission is extended on $\sim 8-10 \mathrm{kpc}$ scales (e.g. Fig. 1; see also Chen et al. 2016, Lang et al. 2019). In comparison, as we showed above, the $870 \mu \mathrm{m}$ dust continuum is much more compact, with an effective radius of just 0 ". $10 \pm 0$ " .04 or $\sim 1 \mathrm{kpc}$. But 

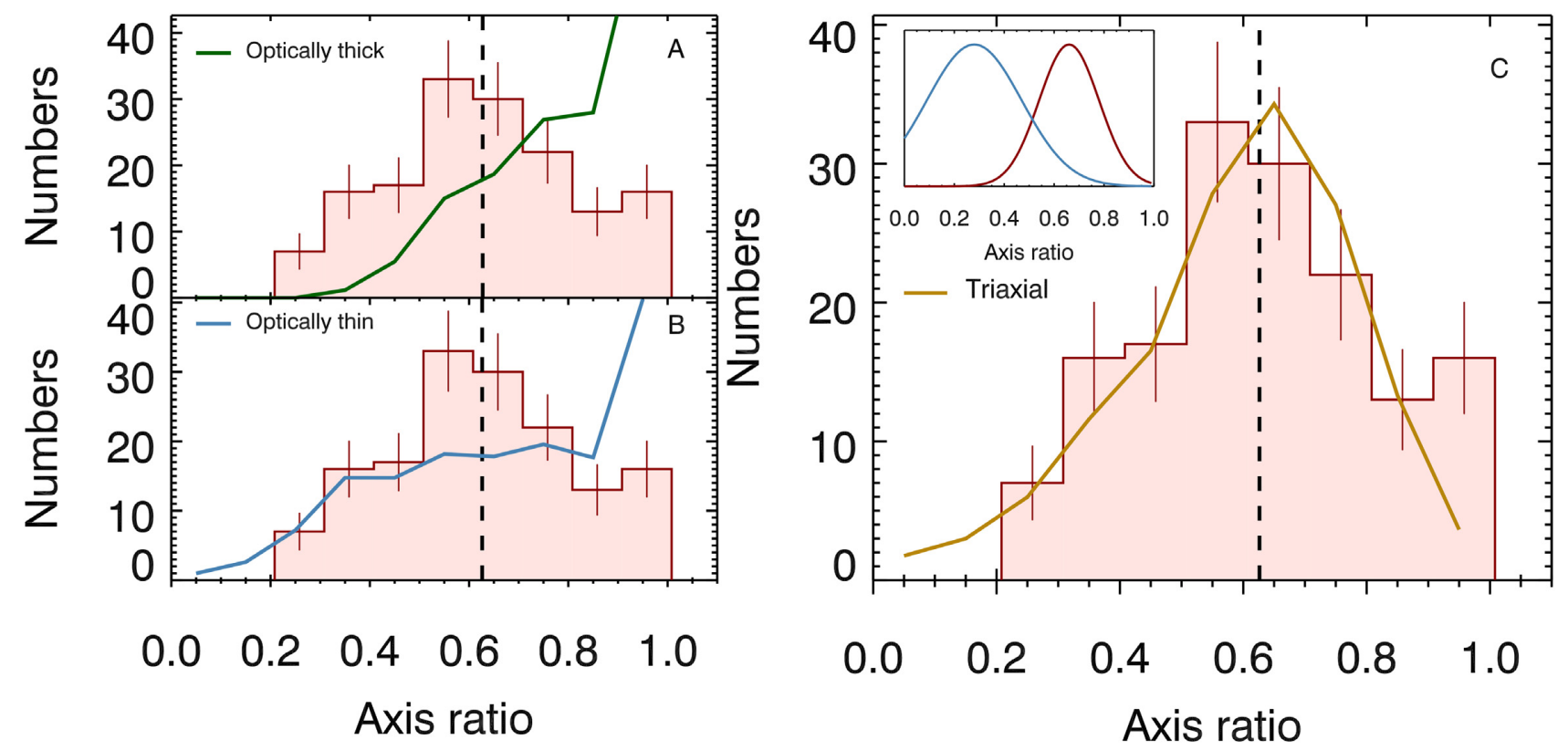

Figure 6. Distribution of the axial ratios for $n=1$ compared with the expected axial ratios from $(a)$ optically thick, $(b)$ optically thin disc models, and $(c)$ from a triaxial model. For the axial ratio distribution of the optically thick and thin models to have a similar 'deficit' of high axial ratios (face-on discs), we would have to assume an unphysical model where we are preferentially viewing the sources at a particular viewing angle $e\left(\sim 40^{\circ}\right)$. In contrast the triaxial model (with a uniform viewing angle distribution) can broadly reproduce the observed axial ratio distribution for our sample of SMGs. Right insert: In the best-fitting triaxial model the intrinsic $b / a$ and $c / a$ distributions that result in the observed axial ratio distributions are Gaussian distributions with a peak at $0.68 \pm 0.02$ and a width of $0.12 \pm 0.06$ for $b / a$ and a peak at $0.28 \pm 0.01$ with a width of $0.19 \pm 0.01$ for $c / a$.

Table 1. Parameter ranges that we apply to explore the triaxial model, and the best-fitting values for the model.

\begin{tabular}{lcc}
\hline Parameter & Full range & Best fit \\
\hline$q_{b / a}$ & $0.1-1$ & $0.66 \pm 0.02$ \\
$\sigma_{b / a}$ & $0.3-3$ & $0.12 \pm 0.06$ \\
$q_{c / a}$ & $0.03-0.3$ & $0.28 \pm 0.01$ \\
$\sigma_{c / a}$ & $0.05-0.5$ & $0.19 \pm 0.01$ \\
\hline
\end{tabular}

is a more extended, lower surface brightness, emission component also present?

Stach et al. (2019) showed that our 0'.18 ALMA resolution observations of the $870 \mu \mathrm{m}$ emission from the SMGs recover $\sim 95$ per cent of the single-dish flux detected with SCUBA-2. This may indicate that a small fraction of the flux is resolved on larger scales, but we do not have the sensitivity to detect this extended emission on a case-by-case basis. Instead to search for this emission we can stack the $870 \mu \mathrm{m}$ continuum maps of the SMGs. As with our size measurements, we perform this stacking in both the visibility and image planes to assess the reliability of our results.

First, we stack the SMGs in the visibility plane, which has the advantage of circumventing issues arising from inhomogeneous beam-sizes of the individual maps. We shift the phase centre of the ALMA primary beam to correspond to the position of the SMG (all of our targets are the sole detected SMGs in their maps) and employ the STACKER library developed for use in CASA to stack the visibilities (Lindroos et al. 2015). The resulting stacked visibilities are then imaged using CASA. Since the SMGs in our sample have a range of flux and signal-to-noise ratio, we stack the data weighted by $1 / \sigma^{2}$. From the stacked visibilities weighted by $1 / \sigma^{2}$, we measure the flux as a function of radius, and show the resulting surface brightness profile as a function of radius in Fig. 7. This figure shows a resolved, high surface brightness central region, but we also clearly detect faint and extended emission on $\geq 0$ '.5 scales, with an integrated flux that is $\sim 10$ per cent of the total flux.

To assess the sensitivity of the derived properties of the extended component on details of the data processing, we also derive a stacked profile by combining the individual image-plane maps of the SMGs for comparison. We extract a 10 arcsec $\times 10$ arcsec thumbnail centred on each SMG, and then average the thumbnails, weighted by $1 / \sigma^{2}$. We then again extract the surface brightness profile from the stacked map and overlay this on to the profile created from the $u v$-stack in Fig. 7. We apply the same procedure to the calibrator, and also overlay this in the same figure. The image-plane- and $u v$-derived stacks are well matched, with both showing the same extended emission on $\geq 0$.'5 scales. We stress that the profile of the (point-source) calibrator, which we scale to the same peak surface brightness, is much narrower than the compact emission and lacks the faint emission halo we see in the SMGs. To test the that this extended emission is not due to weak side-lobes in the individual maps, we use CASA to simulate a source with an $n=1$ light profile and a size and axial ratio equal to that of our sample, but 10 times brighter. This allows us to test if the extended emission we see in the stacked imaged is due to weak emission on large radii from a compact source. This test show that a compact source with $R_{\text {eff }}=0.11$ arcsec observed at 0.18 arcsec resolution does not show emission on $\geq 0.5$ arcsec scale. This imply that the extended emission we detect in the stacked image is indeed likely to be caused by a second component.

To characterize the surface brightness profile, we fit a twocomponent model, including an inner and an outer Sérsic profile each with $n=1$. For the compact component we fix $R_{\text {eff }}$ to the value derived above, $0^{\prime \prime} .10 \pm 0.04$, and for the extended 

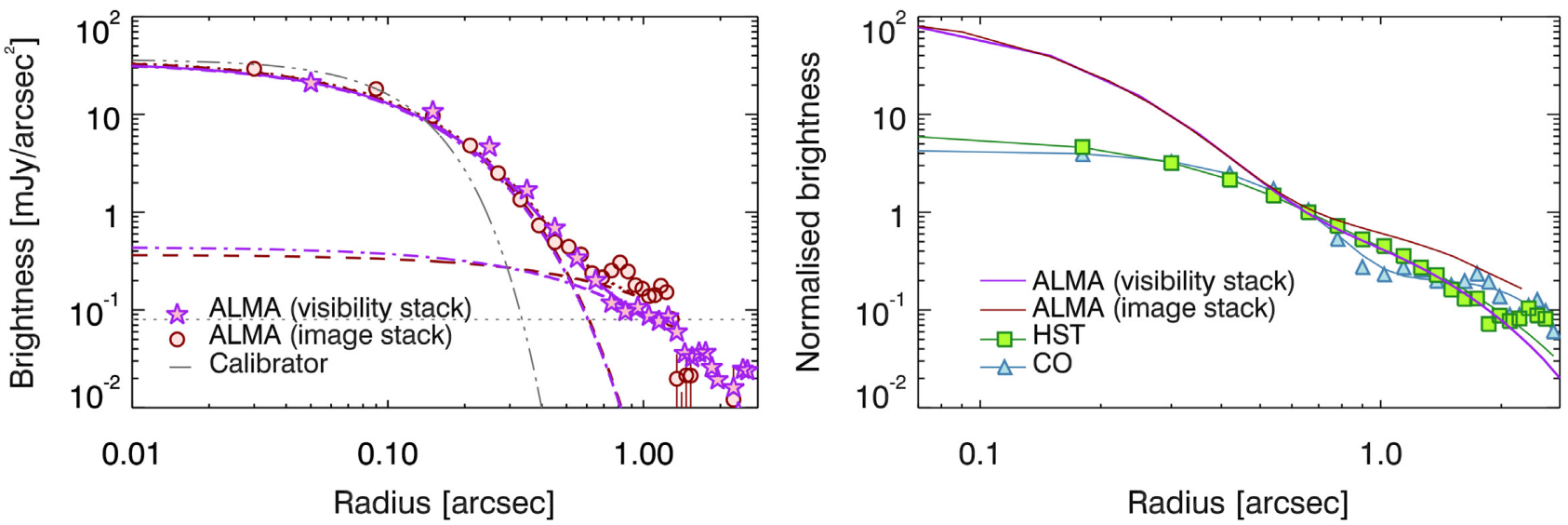

Figure 7. Left: The $870 \mu \mathrm{m}$ continuum light profile obtained by stacking the $870 \mu \mathrm{m}$ dust maps in the visibility and image plane. The profile shows that most of the emission is compact, but that $13 \pm 1$ per cent of the emission is extended on larger scales ( $\geq 0$ '. 5 or $\geq 10 \mathrm{kpc}$ ), and only detected in our maps by stacking. The RMS level of the image stack is illustrated by the dotted line. Right: The $870 \mu \mathrm{m}$ continuum light profile obtained by stacking the $870 \mu \mathrm{m}$ dust maps in the visibility and image planes, normalized and compared to the stacked stellar and molecular gas profile seen in HST and ALMA imaging (Calistro Rivera et al. 2018). While the $870 \mu \mathrm{m}$ dust continuum emission has an excess in the nuclear region compared to the stellar and molecular gas profiles, the extended component follows that of the stellar and molecular gas, suggesting that they trace the same structural component in these systems.

component we adopt $R_{\text {eff }} \sim 0$ ".5. The extended component accounts for $13 \pm 1$ percent of the total emission. This suggest that the SMGs generally comprise a centrally concentrated starburst which accounts for $\sim 90$ per cent of the total dust continuum flux density, with an extended star formation component on scales similar to that seen in the rest-frame UV/optical (as seen by HST). The transition between the compact and extended components occur at $0{ }^{\prime} .15 \pm 00^{\prime} 01(\sim 1.2 \mathrm{kpc}$ at $z \sim 3)$, and the luminosity-weighted average effective radius of the two components, which provide the most appropriate 'size' for the whole systems in 0 ' $15 \pm 0$ '.05 corresponding to $1.2 \pm 0.1 \mathrm{kpc}$. However, in terms of relative surface brightness - the extended component has a peak surface brightness (and hence implied dust mass surface density) which is around two orders of magnitude lower than the compact component.

\section{DISCUSSION}

\subsection{Discs, spheroids, or bars?}

To provide a qualitative context for the structural properties of the SMGs in our sample, we compare in Fig. 8 the observed axial ratio and Sérsic index distributions from the $870 \mu \mathrm{m}$ observations of the SMGs to those derived in the $g$-band for the stellar light distributions of a morphologically classified sample of low-redshift galaxies from the GAMA survey (Kelvin et al. 2014). Consistent with the results from our modelling in the previous section, Fig. 8 shows that the apparent axial ratio distribution of disc galaxies is not a good match to that observed for the SMGs, with a 0 per cent chance that they are drawn from the same parent population. Whereas the SMG distribution is better matched to that of the spheroids, with a KS test returning a 30 per cent chance of the two samples sharing the same parent population.

However, Fig. 8 also shows that spheroids have light profiles which are better described by high Sérsic indexes. This is not the case for the SMGs, which have a median Sérsic index of $n=$ $1.00 \pm 0.12$, with a distribution which differs significantly from the spheroids (a KS test returns a 0 percent probability that the two distributions are from the same parent sample). Whereas the Sérsic index distribution for late-type discs is a much closer match to that seen for the SMGs, with a strong peak at $n \sim 1$, and a tail to higher $n$.

This combination of apparently triaxial structures with exponential surface brightness profiles resembles that seen in the central bars of barred spiral galaxies (e.g. Seigar \& James 1998). This interpretation of the morphology of the dust emission in SMGs was first suggested by Hodge et al. (2019). They re-observed six of the ALESS SMGs from Hodge et al. (2016) with ALMA in deep integrations at $0^{\prime \prime} 07$ resolution and identified complex structures which were unresolved in their earlier 0 '. 15 observations (comparable to the data analysed here). In their higher resolution and deeper maps, Hodge et al. (2019) find symmetric clump-like structures bracketing elongated nuclear emission. They interpret these morphologies as representing bars in galaxies where the 'clump'-like structures are formed through orbit crowding or starforming rings. Hodge et al. (2019) find a ratio of the diameters of the bar-to-ring structures of $1.9 \pm 0.3$ similar to that seen for these components in local barred galaxies. Our results on a larger statistical sample, while lacking the resolution and depth to directly detect these features, have structural properties consistent with the suggestion that much of the dust continuum emission from SMGs arises in bar-like structures in their central regions.

\subsection{What is the extended dust component?}

Our analysis of the stacked profiles of the SMGs in our sample indicates the presence of a spatially-extended component with a peak surface brightness which is roughly two orders of magnitude fainter than the compact component detected in the individual sources, and which contributes $\sim 10$ percent to the total flux densities.

To investigate the relationship between the compact and extended dust continuum emission, we split our sample into four bins of starformation rate (with equal numbers of SMGs in each bin) and stack the maps of these sources in the image plane. We show the resulting surface brightness profiles for the four independent subsamples in Fig. 9. Each of these profiles shows both a compact and an extended component. We fit these with the same double Sérsic 

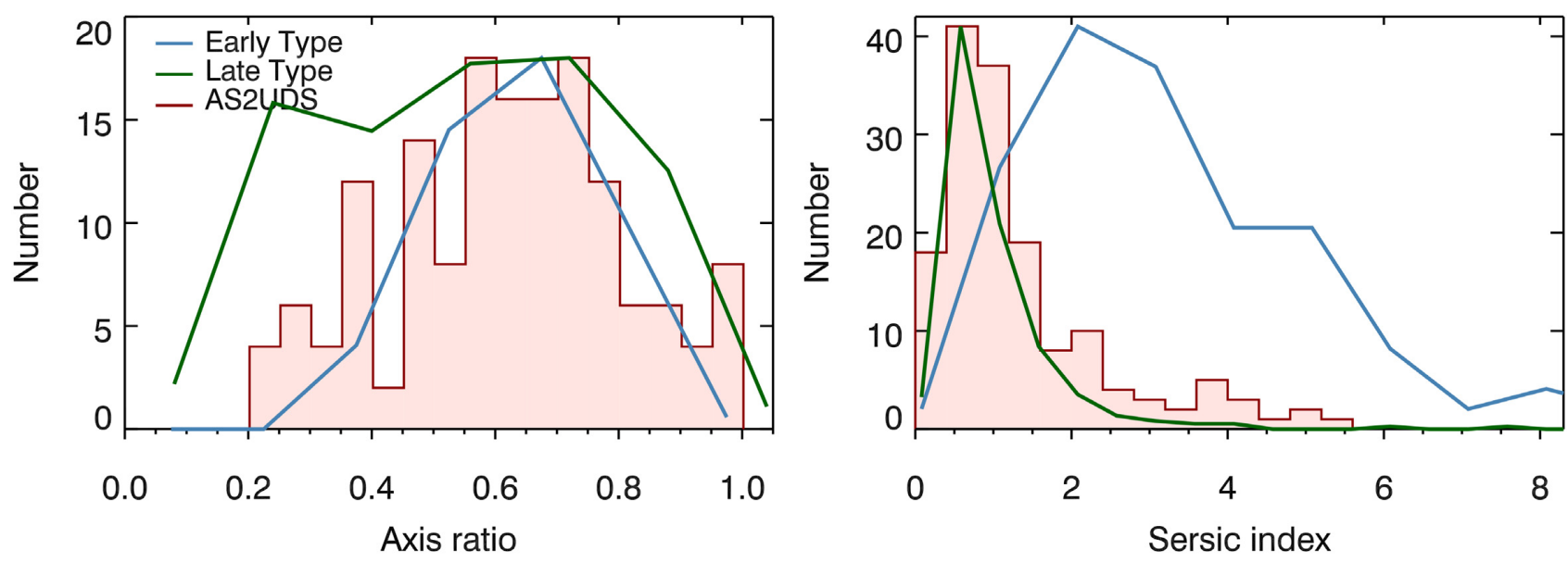

Figure 8. A comparison of the distribution of axial ratios and Sérsic indices for 153 SMGs, to those measured at rest-frame optical wavelengths for morphologically classified late-type discs and early-type spheroidal galaxies from the GAMA survey (Kelvin et al. 2014). Left: Axial ratio distribution of our SMGs compared to that of the late-type disc and spheroids, with the distribution scaled to the peak of our SMG distribution. A KS test shows that our SMG distribution is most similar to that of the spheroidal galaxies, with a 30 per cent chance that these are selected from the same parent sample (compared to 0 per cent match to the late-type discs). This supports the validity of the triaxial model for the morphologies of the SMGs proposed in Section 3.5.1. Right: Sérsic index distribution of our SMGs compared to that of late-type discs and spheroids, now showing a stronger similarity between the SMGs and the late-type disc galaxies, rather than the spheroids (which are rejected as a match based on KS test returning a 0 per cent probability that they are selected from the same parent sample). These two plots thus suggest that the typical $870 \mu \mathrm{m}$ dust emission of our SMGs has a triaxial morphology, but an exponential surface profile - these characteristics are seen for central bars in disc galaxies.
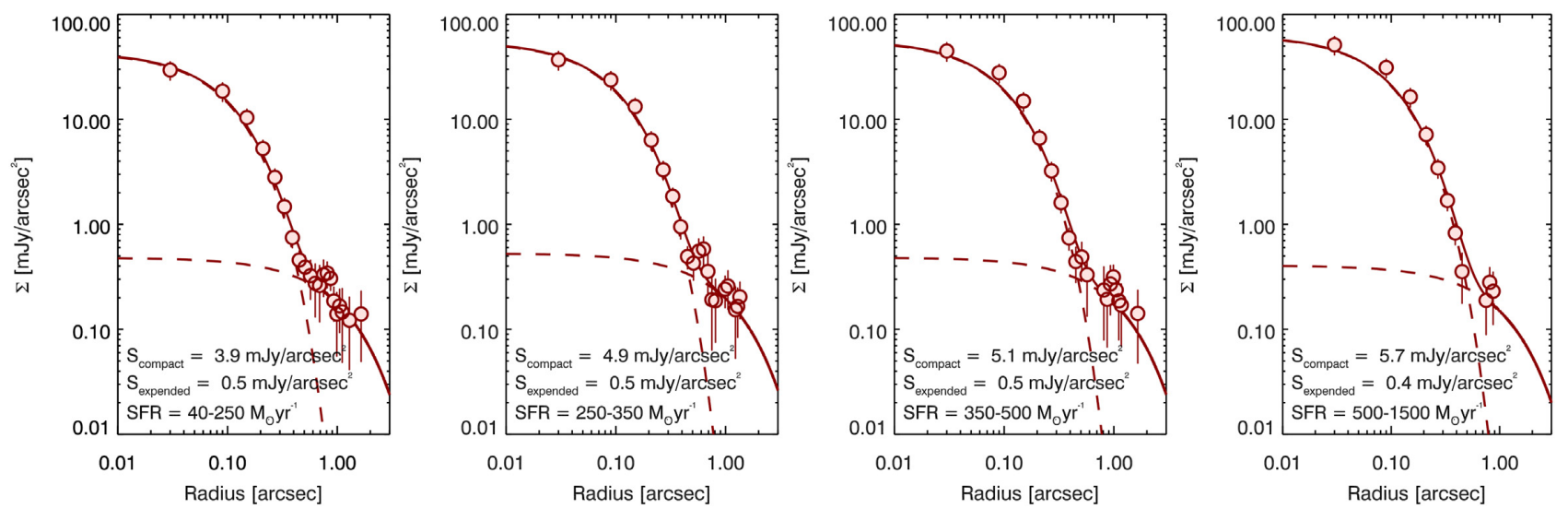

Figure 9. Surface brightness as a function of radius for the image stacks split into bins of star-formation rates of 40-250, 250-350, 350-500, and 500$1500 \mathrm{M}_{\odot} \mathrm{yr}^{-1}$. Both stacks in the image plane and visibility plane return similar profiles, which are composed of a compact and an extended component (we therefore only show the image stack). We fit double exponential profiles and find that the peak flux of the extended component is close to constant at a mean of $0.47 \pm 0.03 \mathrm{mJy}$ for the four stacks, while the compact component becomes brighter with increasing star-formation rate.

model used above to derive the fraction of luminosity in the compact and extended components. We then plot this ratio as a function of star-formation rate in Fig. 10. This figure demonstrates that the luminosity density of the extended emission remains approximately constant, despite the luminosity density of the compact component increasing by a factor of 50 per cent (over a range of a factor $\sim 6$ in total star-formation rate), suggesting that the star formation surface density in the compact and extended regions are decoupled.

We also compare our measurements of the extended component with the median value of a sample of faint field galaxies in strongly lensed cluster in the Hubble Frontier Fields Survey (GonzálezLópez et al. 2017; Laporte et al. 2017). These galaxies at $z \simeq$ 1.0-2.9 are detected with ALMA at $1.1 \mathrm{~mm}$ and represent more typical star-forming field galaxies with star-formation rates of 10$100 \mathrm{M}_{\odot} \mathrm{yr}^{-1}$. Interestingly, the median value for these faint field galaxies show similar surface brightness and extent to the extended components seen in the SMGs, and suggests that the extended star formation we detect in SMGs has similar star formation surface density to that in 'normal' star-forming galaxies in the field. In contrast, the intense star formation surface density in the compact component is significantly higher.

We also wish to understand the relationship of the extended structure we have uncovered at $870 \mu \mathrm{m}$ to the other baryonic components of these systems: the (unobscured) stellar emission and the cool molecular gas. To demonstrate how the observed nearinfrared emission (rest-frame UV/optical at $z \sim 3$ ) compares to the 


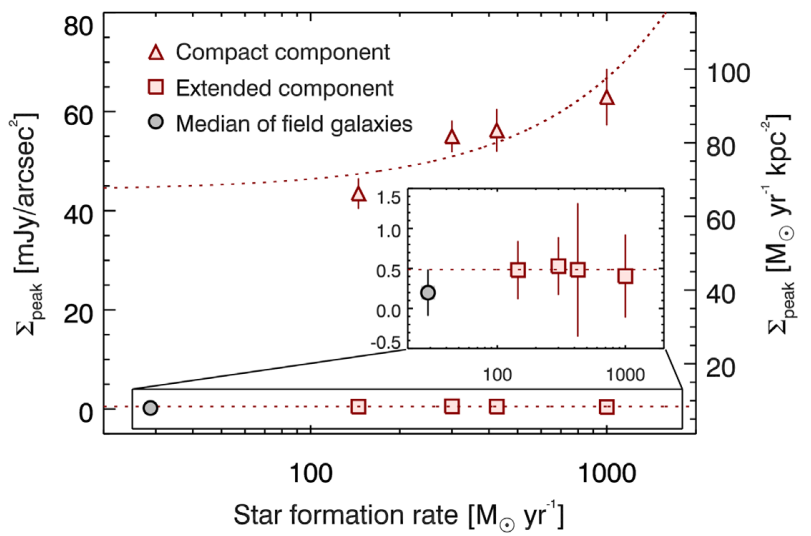

Figure 10. Fitted peak surface brightness as a function of the star-formation rate for the compact and extended components (in Fig. 9). We see that the peak surface brightness for the extended component is roughly constant, while the brightness of the compact component increases as a function of star-formation rate. This is illustrated by the linear fits. We compare the extended components to the surface brightnesses of faint dusty galaxies in the Hubble Frontier Fields, and find a comparable median surface brightness. Hence the extended component we find in the SMGs are comparable in dust surface density to 'normal' star-forming field galaxies.

$870 \mu \mathrm{m}$ emission, in Fig. 7 we overlay the surface brightness profile of the dust continuum emission and the near-infrared from HST (in this figure, we have scaled the surface brightness profiles to the same integral). We also overlay the gas emission as inferred from the molecular ${ }^{12} \mathrm{CO}(3-2)$ emission from four SMGs from ALESS (Calistro Rivera et al. 2018). This low- $J$ CO transition is expected to arise from material in the interstellar medium which has low to moderate critical densities. This means its extent should trace the bulk of the underlying cool gas reservoir in these systems. As can be seen in Fig. 7, the extended emission seen at $870 \mu \mathrm{m}$ from our stack seems to match the spatial extent of the sources as seen in the near-infrared and also in the low- $J$ CO from the molecular gas. Since the extended $870 \mu \mathrm{m}$ dust continuum component follows the same profile as the molecular gas and (less obscured) stellar emission, this suggests that the extended component traces a halo or outer disc in these galaxies, which are dominated by stars and with much lower star formation surface densities and obscuration than the central starbursts.

Similar two-component profiles to that we see in our $870 \mu \mathrm{m}$ stacking analysis have also been observed through stellar-mass surface-density profiles in high-resolution hydrodynamical simulations of merging high-redshift massive starburst discs with properties similar to those of SMGs (Hopkins et al. 2013). The merging galaxies in these simulations are initially disc-dominated, but form nuclear bulges (on kpc scales) dominated by in situ star formation fuelled by gas driven to the centre by strong torques (Mihos \& Hernquist 1994; Hopkins et al. 2008). Using dynamical arguments Hopkins et al. (2009) suggest that because gas can dissipate energy, it can efficiently lose its angular momentum and rapidly fall into the centre in a merger event. This results in a concentrated starburst event seen in, for example, nearby merging ULIRGs and recent merger remnants (e.g. Scoville et al. 1986; Sargent et al. 1987; Sargent, Sanders \& Phillips 1989; Kormendy \& Sanders 1992; Rothberg \& Joseph 2004). This process builds a clear bulge in the centre of the merger remnant.
The extended component or 'envelope' in this simulation is dominated by stars formed before the merger event and gas at large radii with significant angular momentum. This gas does not lose its angular momentum in the merger and re-forms a disc as the remnant relaxes. The survival (or re-formation) of the disc is therefore dependent on how much gas loses its angular momentum (Hopkins et al. 2009). If all the gas in a merger event efficiently loses its angular momentum, it would all be consumed in the nuclear starburst, and no gas would be left to re-form a disc. However, high gas fractions have been shown to be inefficient at losing their angular momentum, leading to the fraction of gas available to fuel the central starburst scaling sub-linearly with the gas fraction, and so leaving gas to re-form a disc (Hopkins et al. 2013).

In the framework of the model developed by Hopkins et al. (2013) we can also investigate the physical nature of the SMGs and their triggering. Besides considering different models with feedback and effective equations of state, Hopkins et al. (2013) also consider the influence of prograde versus retrograde mergers of disc galaxies. The different feedback models and the relative angular momentum vectors of the discs have little influence on the stellar mass profile of the remnant. However, Hopkins et al. (2013) also show that the relative angular momentum vectors and orbit of the merging components has an influence on the timescales with which the merger remnant evolves. A prograde merger develops a morphology with a nuclear starburst and an envelope after $\sim 1$ Gyr, while the morphology of a retrograde merger after $\sim 1$ Gyr still shows two separate disc galaxies. This suggest that if major-merger events are the cause of the nuclear starburst event that we observe for our SMGs, a large fraction will have to be remnants of, or late stage, prograde mergers. If they were retrograde then the median redshift of our sample of $z \sim 2.9$ this means that the two merging disc galaxies would have to have appeared as highly star-forming systems at redshift $\gtrsim 5$. The redshift distribution of SMGs (Chapman et al. 2005; Weiß et al. 2013; Simpson et al. 2014; Dudzevičiūtè et al. 2019) has been shown to peak at $z \sim 2.5-3.5$ and to have a tail out to redshifts $z \sim 7$, meaning that starburst galaxies at $z \gtrsim 5$ do occur, but are rare in $870 \mu \mathrm{m}$-selected samples. Hence we suggest that SMGs at $z \sim$ 2-3 are more likely to be late-stage mergers, rather than merger remnants.

\section{SUMMARY AND CONCLUSIONS}

We analyse the dust continuum morphologies and light profiles of 153 well-detected $(\mathrm{S} / \mathrm{N}>8)$ SMGs observed with ALMA at 0.18 $\operatorname{arcsec}(\sim 1 \mathrm{kpc})$ resolution. We fit both Gaussians (in the visibility and image plane) and free and $n=1$ Sérsic models (in the image plane), and measure the effective radii, axial ratio, and Sérsic indices for the individual sources. We also stack (again in both the visibility and image planes) the $870 \mu \mathrm{m}$ emission for the whole sample and selected subsets to trace fainter and more extended emission around these systems. Our main conclusions are

(i) The median effective radius for SMGs in our sample is $0.10 \pm 0.04$ arcsec. Accounting for the extended dust component we find, we derive a flux-weighted effective radius of $0.15 \pm 0.05$ $\operatorname{arcsec}$ or $1.2 \pm 0.4 \mathrm{kpc}$ at $z \sim 3$. This in consistent with estimates of the sub-millimetre sizes of SMGs from earlier smaller scale studies. We show that there is a rough correlation between the $870 \mu \mathrm{m}$ and observed $K$-band sizes of SMGs in our sample, with the $870 \mu \mathrm{m}$ sizes being on average $2.2 \pm 0.2$ times smaller. 
(ii) The effective radii of SMGs in our sample show a very weak decline with increasing redshifts and a similarly marginal increase with $S_{870}$. Using the physical properties of our sample from Dudzevičiūtè et al. (2019) and assuming a simple source geometry we expect that the typical apparent source size would decrease slightly at higher redshifts, although this evolution would be countered by optical depth effects. The weak decline in size we see with redshift suggests that the physical sizes of SMGs do not evolve strongly with redshift and the lack of variation in size with $S_{870}$ indicates that the more luminous systems are likely to exhibit higher pressures in their interstellar medium.

(iii) We find that the apparent axial ratio distribution of the SMGs is best described by non-axisymmetric morphologies (triaxial) and the Sérsic index distribution has a median of $n=1.0 \pm 0.1$. By comparing these distributions with those of disc and spheroid galaxies, the axial ratio distribution of SMGs is most similar to those of spheroid galaxies, while their Sérsic index distribution is most similar to that of disc galaxies. This combination of exponential surface brightness profiles and triaxial structures are the characteristics of bars in galaxies. Higher resolution and deeper observations of six SMGs by Hodge et al. (2019) have identified potential bar and ring structures in those galaxies and we therefore suggest that the statistical properties of the SMGs in our sample point to bars being a ubiquitous feature of bright SMGs.

(iv) We stack our SMGs in both the image and visibility planes and find that the continuum emission profiles are composed of not only the compact component we have directly detected, but also a much lower surface brightness, extended component. The extended component accounts for $13 \pm 1$ per cent of the total emission and has a scale size of $\sim 0.5 \operatorname{arcsec}(\sim 4 \mathrm{kpc})$ Comparing with stacked $\mathrm{CO}(3-$ 2) and HST imaging of samples of SMGs, we see that the extended component is comparable in size to the low- $J$ CO molecular gas and stellar distributions. We conclude that it is likely that the extended component seen in the stacked $870 \mu \mathrm{m}$ maps traces a surrounding disc or envelope around the central, compact far-infrared luminous starburst.

(v) By stacking the $870 \mu \mathrm{m}$ maps in bins of star-formation rate we find that the size and luminosity of the extended component is roughly constant with total star-formation rate, while the compact component becomes brighter. This suggests that the star formation taking place in the compact component is broadly decoupled from the star formation taking place in the extended component.

We have studied a large sample of SMGs using moderate resolution ALMA data and find that the morphologies observed at 0.18 arcsec resolution are best described by bars in galaxies. However, to confirm this requires deeper observations to detect the extended components in individual maps, higher resolution imaging $(\sim 0.08$ arcsec) to show that the $870 \mu \mathrm{m}$ dust continuum trace bars structures, and ideally dynamical gas measurements of the extended component to determine if this has an order rotational motion as seen for disc or more chaotic as would be expected from a merger event.

\section{ACKNOWLEDGEMENTS}

We thank the anonymous referee for their helpful thorough reading of the manuscript, and suggestions that improved the paper. BG and IRS acknowledge support from the ERC Advanced Investigator program DUSTYGAL (321334). BG, IS, and AMS acknowledge financial support from an STFC grant (ST/P000541/1). JLW acknowledges support from an STFC Ernest Rutherford Fellowship
(ST/P004784/1 and ST/P004784/2). MJM acknowledges the support of the National Science Centre, Poland through the SONATA BIS grant 2018/30/E/ST9/00208. The ALMA data used in this paper were obtained under programs ADS/JAO.ALMA\#2015.1.01528.S. ALMA is a partnership of ESO (representing its member states), NSF (USA), and NINS (Japan), together with NRC (Canada) and NSC and ASIAA (Taiwan), in cooperation with the Republic of Chile. The Joint ALMA Observatory is operated by ESO, AUI/NRAO, and NAOJ.

\section{REFERENCES}

Almaini O. et al., 2017, MNRAS, 472, 1401

Athanassoula E., 2003, MNRAS, 341, 1179

Barger A. J., Cowie L. L., Sanders D. B., Fulton E., Taniguchi Y., Sato Y., Kawara K., Okuda H., 1998, Nature, 394, 248

Battisti A. J. et al., 2019, ApJ, 882, 61

Binney J., Tremaine S., 1987, Galactic Dynamics. Princeton Univ. Press, Princeton, NJ

Bothwell M. S. et al., 2013, MNRAS, 429, 3047

Bournaud F., Elmegreen B. G., 2009, ApJ, 694, L158

Calistro Rivera G. et al., 2018, ApJ, 863, 56

Calzetti D. et al., 2007, ApJ, 666, 870

Chapman S. C., Smail I., Blain A. W., Ivison R. J., 2004, ApJ, 614, 671

Chapman S. C., Blain A. W., Smail I., Ivison R. J., 2005, ApJ, 622, 772

Chen Y., Lowenthal J. D., Yun M. S., 2010, ApJ, 712, 1385

Chen C.-C. et al., 2015, ApJ, 799, 194

Chen C.-C. et al., 2016, ApJ, 831, 91

Clements D. L., Baker A. C., 1996, A\&A, 314, L5

da Cunha E. et al., 2015, ApJ, 806, 110

Dekel A., Sari R., Ceverino D., 2009, ApJ, 703, 785

Dudzevičiūtè U. et al., 2019, preprint (arXiv:1910.07524)

Engel H. et al., 2010, ApJ, 724, 233

Falcón-Barroso J., Knapen J. H., 2013, Secular Evolution of Galaxies. Cambridge Univ. Press, Cambridge

Farrah D. et al., 2001, MNRAS, 326, 1333

Gavazzi G. et al., 2015, A\&A, 580, A116

Geach J. E. et al., 2017, MNRAS, 465, 1789

González-López J. et al., 2017, A\&A, 597, A41

Grogin N. A. et al., 2011, ApJS, 197, 35

Gullberg B. et al., 2018, ApJ, 859, 12

Hodge J. A. et al., 2016, ApJ, 833, 103

Hodge J. A. et al., 2019, ApJ, 876, 130

Hopkins P. F., Hernquist L., Cox T. J., Dutta S. N., Rothberg B., 2008, ApJ, 679,156

Hopkins P. F., Cox T. J., Younger J. D., Hernquist L., 2009, ApJ, 691, 1168

Hopkins P. F., Cox T. J., Hernquist L., Narayanan D., Hayward C. C., Murray N., 2013, MNRAS, 430, 1901

Hughes D. H. et al., 1998, Nature, 394, 241

Ikarashi S. et al., 2015, ApJ, 810, 133

Ikarashi S. et al., 2017, ApJ, 849, L36

Ivison R. J. et al., 2012, MNRAS, 425, 1320

Kelvin L. S. et al., 2014, MNRAS, 439, 1245

Kormendy J., 2013, Secular Evolution in Disk Galaxies. Cambridge Univ. Press, Cambridge, p. 1

Kormendy J., Sanders D. B., 1992, ApJ, 390, L53

Kubo M., Tanaka M., Yabe K., Toft S., Stockmann M., Gómez-Guijarro C., 2018, ApJ, 867, 1

Lang P. et al., 2019, ApJ, 879, 54

Laporte N. et al., 2017, A\&A, 604, A132

Lindroos L., Knudsen K. K., Vlemmings W., Conway J., Martí-Vidal I., 2015, MNRAS, 446, 3502

Lutz D. et al., 2016, A\&A, 591, A136

Lynden-Bell D., 1996, in Sandqvist A., Lindblad P. O., eds, Lecture Notes in Physics, Vol. 474, Barred Galaxies and Circumnuclear Activity. Springer Verlag, Berlin, p. 7 
McAlpine S. et al., 2019, MNRAS, 488, 2440

McMullin J. P., Waters B., Schiebel D., Young W., Golap K., 2007, in Shaw R. A., Hill F., Bell D. J., eds, ASP Conf. Ser. Vol. 376, Astronomical Data Analysis Software and Systems XVI. Astron. Soc. Pac., San Francisco, p. 127

Mihos J. C., Hernquist L., 1994, ApJ, 437, L47

Psychogyios A. et al., 2016, A\&A, 591, A1

Riechers D. A. et al., 2011, ApJ, 733, L12

Robotham A. S. G., Taranu D. S., Tobar R., Moffett A., Driver S. P., 2017, MNRAS, 466, 1513

Rothberg B., Joseph R. D., 2004, AJ, 128, 2098

Ryden B. S., 2004, ApJ, 601, 214

Sakamoto K. et al., 2008, ApJ, 684, 957

Sanders D. B., Mirabel I. F., 1996, ARA\&A, 34, 749

Sargent A. I., Sanders D. B., Scoville N. Z., Soifer B. T., 1987, ApJ, 312, L35

Sargent A. I., Sanders D. B., Phillips T. G., 1989, ApJ, 346, L9

Scoville N. Z., Sanders D. B., Sargent A. I., Soifer B. T., Scott S. L., Lo K. Y., 1986, ApJ, 311, L47

Seigar M. S., James P. A., 1998, MNRAS, 299, 672

Shibuya T., Ouchi M., Harikane Y., 2015, ApJS, 219, 15
Simpson J. M. et al., 2014, ApJ, 788, 125

Simpson J. M. et al., 2015a, ApJ, 799, 81

Simpson J. M. et al., 2015b, ApJ, 807, 128

Simpson J. M. et al., 2017, ApJ, 839, 58

Smail I., Ivison R. J., Blain A. W., 1997, ApJ, 490, L5

Spergel D. N. et al., 2007, ApJS, 170, 377

Stach S. M. et al., 2019, MNRAS, 487, 4648

Surace J. A., Sanders D. B., Evans A. S., 2001, AJ, 122, 2791

Swinbank A. M. et al., 2011, ApJ, 742, 11

Swinbank A. M. et al., 2015, ApJ, 806, L17

Tacconi L. J. et al., 2008, ApJ, 680, 246

Tadaki K.-i. et al., 2017, ApJ, 834, 135

van der Wel A. et al., 2014, ApJ, 788, 28

Veilleux S., 2002, in Mulchaey J. S., Stocke J. T., eds, ASP Conf. Ser

Vol. 254, Extragalactic Gas at Low Redshift. Astron. Soc. Pac., San Francisco, p. 313

Weiß A. et al., 2013, ApJ, 767, 88

\section{APPENDIX}



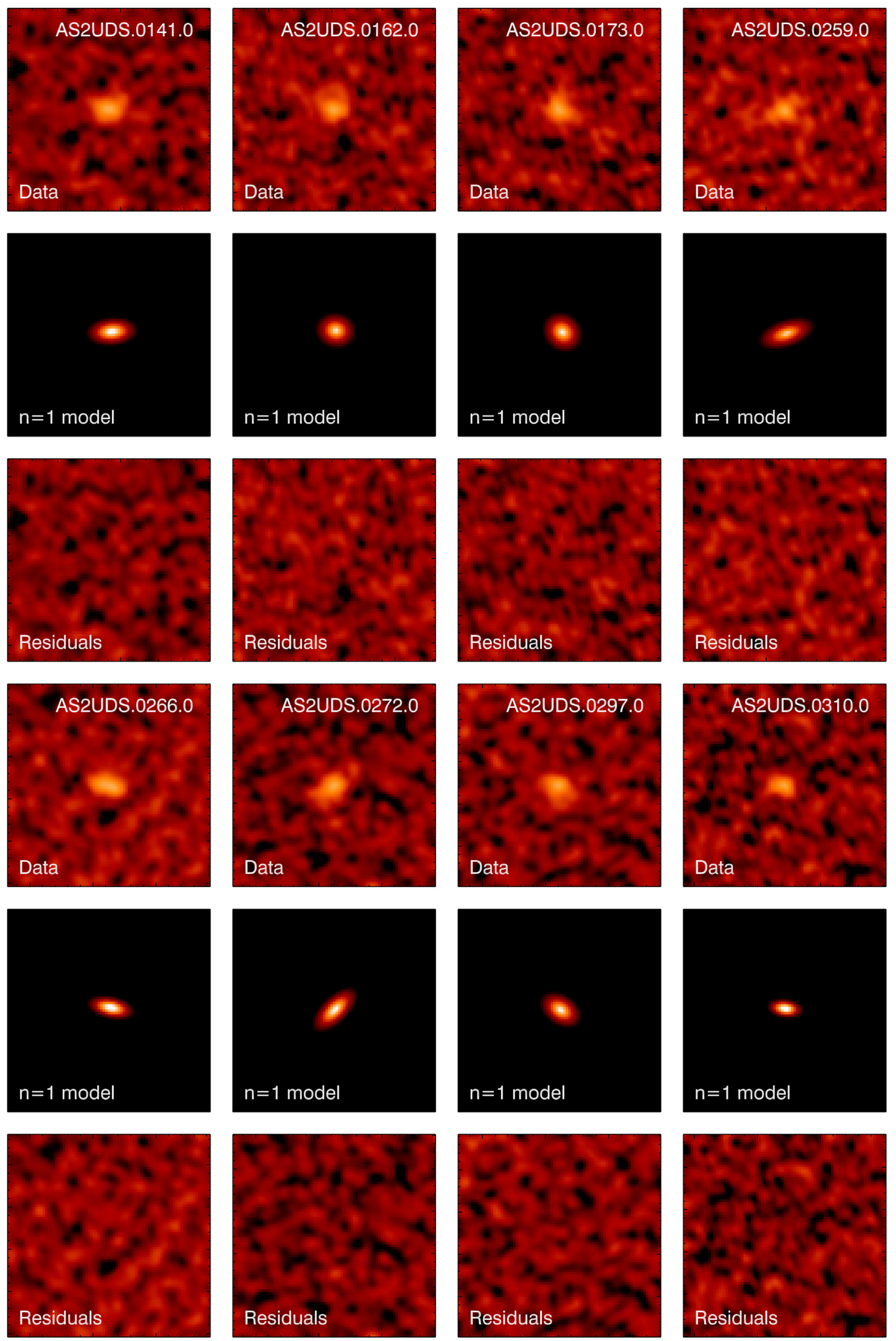

Figure A1. Examples of Sérsic fits with $n=1$ to the eight examples shown in Fig. 1. The rows show the data, model, and the residuals. 
Table A1. The parameters for the $870 \mu \mathrm{m}$ continuum maps measured using Sérsic with a fixed $n=1$ and a free Sersic fit in the image plane. Column 2: photometric redshifts from Dudzevičiūte et al. (2019) with the upper and lower limits of the redshifts given in the power and subscript, Column 3: The star-formation rate (SFR) from Dudzevičiūte et al. (2019), Column 4: The circularized effective radius from the fixed $n=1$ Sérsic fit, Column 5: The circularized effective radius from the Free Sérsic fit, Column 6: The axial ratio from the fixed $n=1$ fit, Column 7: The position angle from the fixed $n=1$ fit, Column 8: The Sérsic fit from the free Sérsic fit.

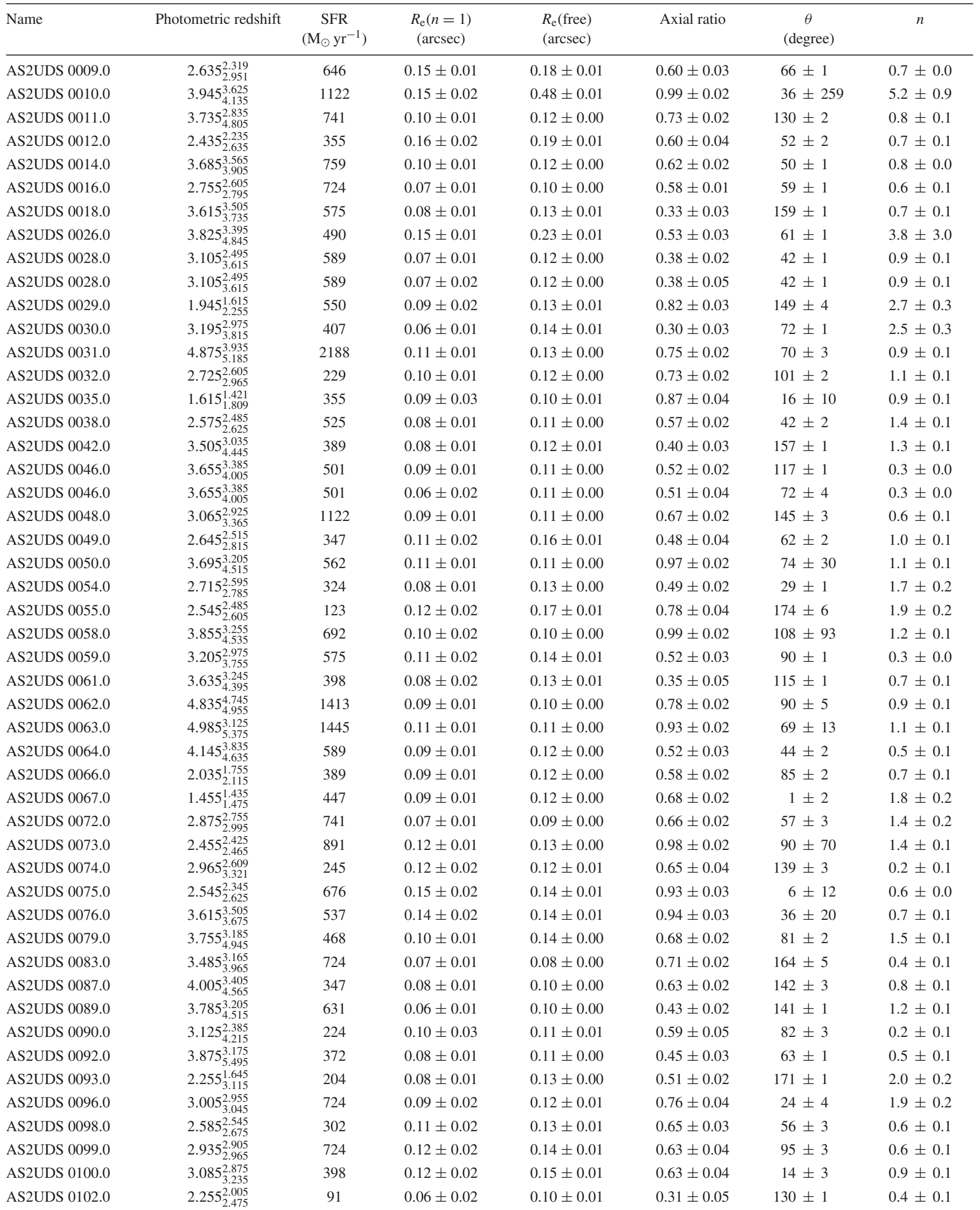


Table A1 - continued

\begin{tabular}{|c|c|c|c|c|c|c|c|}
\hline Name & Photometric redshift & $\begin{array}{c}\mathrm{SFR} \\
\left(\mathrm{M}_{\odot} \mathrm{yr}^{-1}\right)\end{array}$ & $\begin{array}{c}R_{\mathrm{e}}(n=1) \\
(\operatorname{arcsec})\end{array}$ & $\begin{array}{l}R_{\mathrm{e}}(\text { free }) \\
(\operatorname{arcsec})\end{array}$ & Axial ratio & $\begin{array}{c}\theta \\
\text { (degree) }\end{array}$ & $n$ \\
\hline AS2UDS 0103.0 & $3.075_{3.115}^{3.055}$ & 501 & $0.07 \pm 0.01$ & $0.10 \pm 0.00$ & $0.39 \pm 0.03$ & $73 \pm 1$ & $0.2 \pm 0.1$ \\
\hline AS2UDS 0107.0 & $3.015_{3.085}^{2.965}$ & 562 & $0.08 \pm 0.01$ & $0.10 \pm 0.00$ & $0.76 \pm 0.02$ & $64 \pm 3$ & $1.0 \pm 0.1$ \\
\hline AS2UDS 0110.0 & $3.505_{3.585}^{3.385}$ & 1202 & $0.10 \pm 0.02$ & $0.23 \pm 0.01$ & $0.82 \pm 0.03$ & $25 \pm 8$ & $4.2 \pm 0.8$ \\
\hline AS2UDS 0112.0 & $1.805_{2.605}^{1.335}$ & 229 & $0.09 \pm 0.01$ & $0.11 \pm 0.00$ & $0.71 \pm 0.02$ & $87 \pm 3$ & $1.0 \pm 0.1$ \\
\hline AS2UDS 0124.0 & $3.665_{4.675}^{3.045}$ & 331 & $0.10 \pm 0.02$ & $0.12 \pm 0.01$ & $0.68 \pm 0.03$ & $176 \pm 3$ & $0.9 \pm 0.1$ \\
\hline AS2UDS 0126.0 & $2.575_{3.315}^{1.955}$ & 676 & $0.09 \pm 0.02$ & $0.11 \pm 0.01$ & $0.60 \pm 0.04$ & $141 \pm 2$ & $0.7 \pm 0.1$ \\
\hline AS2UDS 0128.0 & $3.265_{4.155}^{2.955}$ & 257 & $0.10 \pm 0.02$ & $0.15 \pm 0.01$ & $0.50 \pm 0.04$ & $54 \pm 2$ & $1.2 \pm 0.1$ \\
\hline AS2UDS 0132.0 & $3.075_{3.215}^{3.015}$ & 372 & $0.07 \pm 0.01$ & $0.07 \pm 0.00$ & $0.85 \pm 0.02$ & $55 \pm 8$ & $1.4 \pm 0.2$ \\
\hline AS2UDS 0135.0 & $3.615_{5.145}^{3.175}$ & 708 & $0.10 \pm 0.01$ & $0.17 \pm 0.00$ & $0.77 \pm 0.02$ & $71 \pm 3$ & $2.7 \pm 0.2$ \\
\hline AS2UDS 0147.0 & $2.175_{2.825}^{1.535}$ & 200 & $0.06 \pm 0.01$ & $0.08 \pm 0.00$ & $0.68 \pm 0.02$ & $54 \pm 4$ & $0.8 \pm 0.2$ \\
\hline AS2UDS 0149.0 & $3.065_{3.205}^{2.975}$ & 316 & $0.04 \pm 0.01$ & $0.09 \pm 0.00$ & $0.21 \pm 0.04$ & $16 \pm 1$ & $1.0 \pm 0.1$ \\
\hline AS2UDS 0151.0 & $2.995_{3.175}^{2.785}$ & 501 & $0.08 \pm 0.01$ & $0.10 \pm 0.00$ & $0.64 \pm 0.02$ & $164 \pm 3$ & $0.7 \pm 0.1$ \\
\hline AS2UDS 0158.0 & $2.965_{3.115}^{2.585}$ & 398 & $0.08 \pm 0.01$ & $0.08 \pm 0.00$ & $1.00 \pm 0.02$ & $144 \pm * * *$ & $0.5 \pm 0.1$ \\
\hline AS2UDS 0162.0 & $3.765_{5.165}^{3.155}$ & 355 & $0.11 \pm 0.02$ & $0.12 \pm 0.01$ & $0.87 \pm 0.03$ & $79 \pm 11$ & $0.7 \pm 0.1$ \\
\hline AS2UDS 0164.0 & $3.135_{5.145}^{2.665}$ & 339 & $0.10 \pm 0.02$ & $0.14 \pm 0.01$ & $0.78 \pm 0.04$ & $130 \pm 6$ & $2.0 \pm 0.3$ \\
\hline AS2UDS 0165.0 & $2.255_{2.565}^{1.945}$ & 603 & $0.04 \pm 0.01$ & $0.09 \pm 0.00$ & $0.23 \pm 0.03$ & $114 \pm 1$ & $0.7 \pm 0.1$ \\
\hline AS2UDS 0166.0 & $2.305_{2.315}^{2.295}$ & 309 & $0.05 \pm 0.01$ & $0.09 \pm 0.00$ & $0.33 \pm 0.02$ & $64 \pm 1$ & $0.6 \pm 0.1$ \\
\hline AS2UDS 0167.0 & $2.685_{3.007}^{2.363}$ & 479 & $0.09 \pm 0.02$ & $0.11 \pm 0.01$ & $0.64 \pm 0.03$ & $154 \pm 3$ & $0.8 \pm 0.1$ \\
\hline AS2UDS 0169.0 & $3.595_{4.065}^{3.255}$ & 575 & $0.11 \pm 0.02$ & $0.21 \pm 0.01$ & $0.52 \pm 0.05$ & $165 \pm 2$ & $4.8 \pm 3.0$ \\
\hline AS2UDS 0185.0 & $5.325_{6.375}^{3.235}$ & 575 & $0.10 \pm 0.02$ & $0.11 \pm 0.00$ & $0.87 \pm 0.02$ & $137 \pm 8$ & $0.5 \pm 0.1$ \\
\hline AS2UDS 0187.0 & $1.945_{2.135}^{1.775}$ & 200 & $0.08 \pm 0.02$ & $0.10 \pm 0.01$ & $0.65 \pm 0.03$ & $116 \pm 3$ & $0.3 \pm 0.1$ \\
\hline AS2UDS 0192.0 & $3.375_{4.185}^{3.185}$ & 316 & $0.14 \pm 0.02$ & $0.15 \pm 0.01$ & $0.96 \pm 0.03$ & $18 \pm 27$ & $1.3 \pm 0.1$ \\
\hline AS2UDS 0199.0 & $2.985_{3.005}^{2.965}$ & 407 & $0.05 \pm 0.02$ & $0.09 \pm 0.01$ & $0.28 \pm 0.04$ & $168 \pm 1$ & $0.9 \pm 0.1$ \\
\hline AS2UDS 0203.0 & $1.855_{2.078}^{1.632}$ & 214 & $0.11 \pm 0.02$ & $0.15 \pm 0.01$ & $0.58 \pm 0.04$ & $58 \pm 2$ & $1.1 \pm 0.1$ \\
\hline AS2UDS 0205.0 & $3.535_{4.335}^{3.085}$ & 389 & $0.08 \pm 0.01$ & $0.10 \pm 0.00$ & $0.63 \pm 0.02$ & $25 \pm 2$ & $1.2 \pm 0.1$ \\
\hline AS2UDS 0209.0 & $3.595_{3.685}^{3.585}$ & 355 & $0.10 \pm 0.02$ & $0.20 \pm 0.01$ & $0.67 \pm 0.03$ & $56 \pm 4$ & $2.9 \pm 0.4$ \\
\hline AS2UDS 0210.0 & $2.275_{2.395}^{2.175}$ & 214 & $0.09 \pm 0.01$ & $0.11 \pm 0.00$ & $0.63 \pm 0.02$ & $161 \pm 3$ & $1.2 \pm 0.1$ \\
\hline AS2UDS 0212.0 & $2.595_{2.955}^{2.575}$ & 209 & $0.09 \pm 0.02$ & $0.11 \pm 0.01$ & $0.58 \pm 0.04$ & $135 \pm 3$ & $0.6 \pm 0.1$ \\
\hline AS2UDS 0213.0 & $2.825_{3.025}^{2.625}$ & 347 & $0.12 \pm 0.02$ & $0.17 \pm 0.01$ & $0.52 \pm 0.04$ & $40 \pm 2$ & $1.4 \pm 0.1$ \\
\hline AS2UDS 0218.0 & $2.985_{3.515}^{2.655}$ & 224 & $0.05 \pm 0.02$ & $0.22 \pm 0.01$ & $0.24 \pm 0.06$ & $117 \pm 1$ & $4.2 \pm 3.0$ \\
\hline AS2UDS 0222.0 & $3.665_{4.275}^{3.255}$ & 776 & $0.05 \pm 0.01$ & $0.07 \pm 0.00$ & $0.62 \pm 0.01$ & $22 \pm 2$ & $3.6 \pm 0.7$ \\
\hline AS2UDS 0225.0 & $3.375_{4.535}^{2.895}$ & 234 & $0.07 \pm 0.02$ & $0.10 \pm 0.01$ & $0.45 \pm 0.03$ & $61 \pm 2$ & $0.6 \pm 0.1$ \\
\hline AS2UDS 0226.0 & $2.225_{2.255}^{2.195}$ & 209 & $0.06 \pm 0.02$ & $0.10 \pm 0.01$ & $0.52 \pm 0.03$ & $12 \pm 3$ & $2.3 \pm 0.5$ \\
\hline AS2UDS 0231.0 & $2.955_{3.075}^{2.875}$ & 347 & $0.09 \pm 0.02$ & $0.19 \pm 0.01$ & $0.33 \pm 0.07$ & $140 \pm 1$ & $1.7 \pm 0.2$ \\
\hline AS2UDS 0232.0 & $2.525_{2.705}^{2.235}$ & 240 & $0.08 \pm 0.01$ & $0.08 \pm 0.00$ & $0.95 \pm 0.02$ & $110 \pm 28$ & $1.1 \pm 0.2$ \\
\hline AS2UDS 0235.0 & $4.345_{5.815}^{3.395}$ & 380 & $0.10 \pm 0.02$ & $0.12 \pm 0.01$ & $0.67 \pm 0.04$ & $83 \pm 4$ & $1.2 \pm 0.2$ \\
\hline AS2UDS 0236.0 & $3.945_{4.875}^{3.205}$ & 288 & $0.07 \pm 0.02$ & $0.10 \pm 0.01$ & $0.44 \pm 0.04$ & $138 \pm 2$ & $0.4 \pm 0.1$ \\
\hline AS2UDS 0238.0 & $2.175_{2.285}^{2.105}$ & 178 & $0.11 \pm 0.02$ & $0.10 \pm 0.01$ & $0.99 \pm 0.03$ & $109 \pm 111$ & $0.7 \pm 0.1$ \\
\hline AS2UDS 0243.0 & $1.775_{1.785}^{1.765}$ & 1072 & $0.06 \pm 0.02$ & $0.07 \pm 0.01$ & $0.66 \pm 0.03$ & $104 \pm 5$ & $0.2 \pm 0.2$ \\
\hline
\end{tabular}


Table A1 - continued

\begin{tabular}{|c|c|c|c|c|c|c|c|}
\hline Name & Photometric redshift & $\begin{array}{c}\mathrm{SFR} \\
\left(\mathrm{M}_{\odot} \mathrm{yr}^{-1}\right)\end{array}$ & $\begin{array}{c}R_{\mathrm{e}}(n=1) \\
\quad(\operatorname{arcsec})\end{array}$ & $\begin{array}{l}R_{\mathrm{e}}(\text { free }) \\
(\operatorname{arcsec})\end{array}$ & Axial ratio & $\begin{array}{c}\theta \\
\text { (degree) }\end{array}$ & $n$ \\
\hline AS2UDS 0265.0 & $2.295_{2.325}^{2.185}$ & 245 & $0.04 \pm 0.01$ & $0.06 \pm 0.00$ & $0.37 \pm 0.02$ & $103 \pm 2$ & $1.1 \pm 0.2$ \\
\hline AS2UDS 0266.0 & $2.745_{3.015}^{2.515}$ & 145 & $0.06 \pm 0.02$ & $0.06 \pm 0.00$ & $0.47 \pm 0.03$ & $77 \pm 2$ & $0.2 \pm 0.1$ \\
\hline AS2UDS 0269.0 & $2.545_{2.575}^{2.505}$ & 380 & $0.05 \pm 0.01$ & $0.07 \pm 0.00$ & $0.58 \pm 0.02$ & $113 \pm 3$ & $1.0 \pm 0.2$ \\
\hline AS2UDS 0272.0 & $1.775_{1.988}^{1.562}$ & 380 & $0.08 \pm 0.01$ & $0.12 \pm 0.01$ & $0.46 \pm 0.03$ & $135 \pm 2$ & $0.7 \pm 0.1$ \\
\hline AS2UDS 0278.0 & $2.495_{2.545}^{2.435}$ & 316 & $0.06 \pm 0.01$ & $0.06 \pm 0.00$ & $0.70 \pm 0.02$ & $128 \pm 4$ & $0.2 \pm 0.2$ \\
\hline AS2UDS 0280.0 & $2.685_{2.885}^{2.575}$ & 646 & $0.06 \pm 0.02$ & $0.12 \pm 0.01$ & $0.52 \pm 0.04$ & $83 \pm 4$ & $2.1 \pm 0.3$ \\
\hline AS2UDS 0286.0 & $3.235_{3.395}^{3.085}$ & 339 & $0.09 \pm 0.02$ & $0.12 \pm 0.01$ & $0.72 \pm 0.03$ & $17 \pm 5$ & $1.7 \pm 0.3$ \\
\hline AS2UDS 0297.0 & $1.675_{1.876}^{1.474}$ & 316 & $0.09 \pm 0.02$ & $0.11 \pm 0.01$ & $0.67 \pm 0.03$ & $55 \pm 4$ & $0.7 \pm 0.1$ \\
\hline AS2UDS 0298.0 & $2.475_{2.555}^{2.335}$ & 209 & $0.07 \pm 0.02$ & $0.24 \pm 0.02$ & $0.40 \pm 0.04$ & $82 \pm 2$ & $4.0 \pm 0.9$ \\
\hline AS2UDS 0302.0 & $3.665_{4.505}^{3.055}$ & 316 & $0.08 \pm 0.02$ & $0.11 \pm 0.01$ & $0.56 \pm 0.03$ & $88 \pm 2$ & $1.3 \pm 0.2$ \\
\hline AS2UDS 0306.0 & $1.535_{1.585}^{1.505}$ & 41 & $0.08 \pm 0.02$ & $0.13 \pm 0.01$ & $0.62 \pm 0.04$ & $80 \pm 4$ & $2.3 \pm 0.5$ \\
\hline AS2UDS 0310.0 & $3.305_{4.125}^{2.835}$ & 240 & $0.05 \pm 0.02$ & $0.07 \pm 0.01$ & $0.54 \pm 0.04$ & $82 \pm 4$ & $0.2 \pm 0.2$ \\
\hline AS2UDS 0315.0 & $3.305_{4.505}^{2.945}$ & 339 & $0.10 \pm 0.02$ & $0.25 \pm 0.01$ & $0.75 \pm 0.03$ & $167 \pm 5$ & $3.8 \pm 0.7$ \\
\hline AS2UDS 0316.0 & $3.395_{3.565}^{3.235}$ & 646 & $0.10 \pm 0.02$ & $0.20 \pm 0.01$ & $0.53 \pm 0.05$ & $36 \pm 3$ & $2.9 \pm 3.0$ \\
\hline AS2UDS 0321.0 & $2.765_{2.805}^{2.665}$ & 955 & $0.07 \pm 0.02$ & $0.10 \pm 0.01$ & $0.59 \pm 0.03$ & $12 \pm 3$ & $0.3 \pm 0.1$ \\
\hline AS2UDS 0325.0 & $3.425_{4.115}^{3.085}$ & 490 & $0.07 \pm 0.02$ & $0.10 \pm 0.01$ & $0.46 \pm 0.04$ & $74 \pm 2$ & $0.9 \pm 0.2$ \\
\hline AS2UDS 0331.0 & $2.455_{2.715}^{2.155}$ & 219 & $0.05 \pm 0.01$ & $0.05 \pm 0.00$ & $0.88 \pm 0.02$ & $133 \pm 23$ & $2.3 \pm 0.9$ \\
\hline AS2UDS 0336.0 & $5.185_{5.525}^{3.025}$ & 724 & $0.08 \pm 0.02$ & $0.11 \pm 0.01$ & $0.66 \pm 0.03$ & $119 \pm 3$ & $1.3 \pm 0.2$ \\
\hline AS2UDS 0343.0 & $3.275_{4.545}^{2.665}$ & 331 & $0.08 \pm 0.01$ & $0.27 \pm 0.01$ & $0.57 \pm 0.02$ & $64 \pm 2$ & $5.2 \pm 0.9$ \\
\hline AS2UDS 0347.0 & $2.655_{3.515}^{1.895}$ & 331 & $0.11 \pm 0.02$ & $0.14 \pm 0.01$ & $0.64 \pm 0.04$ & $51 \pm 3$ & $1.1 \pm 0.1$ \\
\hline AS2UDS 0348.0 & $3.405_{3.545}^{3.235}$ & 427 & $0.07 \pm 0.02$ & $0.09 \pm 0.00$ & $0.80 \pm 0.02$ & $73 \pm 8$ & $3.6 \pm 0.9$ \\
\hline AS2UDS 0353.0 & $2.625_{2.685}^{2.565}$ & 204 & $0.06 \pm 0.02$ & $0.10 \pm 0.01$ & $0.41 \pm 0.04$ & $137 \pm 2$ & $2.0 \pm 0.4$ \\
\hline AS2UDS 0368.0 & $3.735_{4.215}^{3.245}$ & 617 & $0.08 \pm 0.01$ & $0.11 \pm 0.01$ & $0.54 \pm 0.03$ & $43 \pm 2$ & $1.5 \pm 0.2$ \\
\hline AS2UDS 0374.0 & $2.785_{2.965}^{2.545}$ & 372 & $0.09 \pm 0.01$ & $0.11 \pm 0.00$ & $0.70 \pm 0.03$ & $3 \pm 3$ & $0.9 \pm 0.1$ \\
\hline AS2UDS 0389.0 & $2.615_{2.995}^{2.395}$ & 575 & $0.06 \pm 0.01$ & $0.11 \pm 0.01$ & $0.41 \pm 0.03$ & $12 \pm 2$ & $1.8 \pm 0.2$ \\
\hline AS2UDS 0395.0 & $2.345_{2.626}^{2.064}$ & 204 & $0.06 \pm 0.01$ & $0.07 \pm 0.00$ & $0.78 \pm 0.02$ & $30 \pm 6$ & $1.0 \pm 0.2$ \\
\hline AS2UDS 0402.0 & $2.575_{2.885}^{2.375}$ & 186 & $0.06 \pm 0.02$ & $0.07 \pm 0.01$ & $0.89 \pm 0.03$ & $14 \pm 18$ & $0.9 \pm 0.3$ \\
\hline AS2UDS 0403.0 & $3.015_{3.145}^{2.815}$ & 316 & $0.08 \pm 0.02$ & $0.10 \pm 0.01$ & $0.57 \pm 0.03$ & $103 \pm 3$ & $0.3 \pm 0.1$ \\
\hline AS2UDS 0413.0 & $1.635_{1.655}^{1.615}$ & 204 & $0.07 \pm 0.02$ & $0.09 \pm 0.01$ & $0.54 \pm 0.03$ & $151 \pm 3$ & $1.0 \pm 0.2$ \\
\hline AS2UDS 0432.0 & $2.515_{2.885}^{2.375}$ & 309 & $0.04 \pm 0.01$ & $0.07 \pm 0.00$ & $0.39 \pm 0.03$ & $39 \pm 2$ & $1.0 \pm 0.2$ \\
\hline AS2UDS 0438.0 & $3.445_{4.785}^{2.995}$ & 347 & $0.09 \pm 0.02$ & $0.12 \pm 0.01$ & $0.47 \pm 0.05$ & $43 \pm 2$ & $0.4 \pm 0.1$ \\
\hline AS2UDS 0439.0 & $3.865_{5.505}^{3.165}$ & 347 & $0.09 \pm 0.02$ & $0.09 \pm 0.01$ & $0.95 \pm 0.03$ & $131 \pm 27$ & $1.1 \pm 0.2$ \\
\hline AS2UDS 0440.0 & $1.985_{2.055}^{1.815}$ & 195 & $0.12 \pm 0.02$ & $0.14 \pm 0.01$ & $0.78 \pm 0.03$ & $137 \pm 4$ & $0.8 \pm 0.1$ \\
\hline AS2UDS 0444.0 & $2.555_{2.735}^{2.325}$ & 145 & $0.08 \pm 0.02$ & $0.10 \pm 0.01$ & $0.73 \pm 0.03$ & $165 \pm 5$ & $1.2 \pm 0.2$ \\
\hline AS2UDS 0447.0 & $1.725_{1.835}^{1.705}$ & 407 & $0.10 \pm 0.02$ & $0.10 \pm 0.01$ & $0.92 \pm 0.03$ & $72 \pm 21$ & $1.1 \pm 0.2$ \\
\hline AS2UDS 0454.0 & $3.525_{4.315}^{3.005}$ & 288 & $0.04 \pm 0.01$ & $0.04 \pm 0.00$ & $0.49 \pm 0.02$ & $86 \pm 3$ & $0.2 \pm 0.2$ \\
\hline AS2UDS 0462.0 & 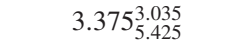 & 372 & $0.12 \pm 0.03$ & $0.23 \pm 0.01$ & $0.61 \pm 0.05$ & $97 \pm 3$ & $2.4 \pm 0.3$ \\
\hline AS2UDS 0465.0 & $2.375_{2.395}^{2.335}$ & 676 & $0.07 \pm 0.01$ & $0.07 \pm 0.00$ & $0.92 \pm 0.02$ & $143 \pm 14$ & $1.4 \pm 0.2$ \\
\hline AS2UDS 0470.0 & $3.275_{3.565}^{2.975}$ & 562 & $0.06 \pm 0.01$ & $0.05 \pm 0.00$ & $1.00 \pm 0.02$ & $144 \pm * * *$ & $0.8 \pm 0.3$ \\
\hline AS2UDS 0481.0 & $3.025_{3.175}^{2.915}$ & 145 & $0.13 \pm 0.02$ & $0.14 \pm 0.01$ & $0.84 \pm 0.04$ & $91 \pm 9$ & $1.1 \pm 0.1$ \\
\hline AS2UDS 0483.0 & $1.855_{2.185}^{1.525}$ & 166 & $0.06 \pm 0.02$ & $0.12 \pm 0.01$ & $0.50 \pm 0.04$ & $161 \pm 3$ & $2.9 \pm 0.8$ \\
\hline AS2UDS 0484.0 & $2.845_{3.635}^{2.175}$ & 398 & $0.04 \pm 0.01$ & $0.07 \pm 0.00$ & $0.35 \pm 0.03$ & $166 \pm 2$ & $1.2 \pm 0.2$ \\
\hline AS2UDS 0487.0 & $3.405_{3.575}^{3.275}$ & 603 & $0.05 \pm 0.02$ & $0.06 \pm 0.01$ & $0.50 \pm 0.04$ & $169 \pm 3$ & $0.2 \pm 0.1$ \\
\hline AS2UDS 0489.0 & $2.205_{2.465}^{1.555}$ & 331 & $0.06 \pm 0.02$ & $0.11 \pm 0.01$ & $0.43 \pm 0.05$ & $132 \pm 3$ & $2.2 \pm 0.4$ \\
\hline AS2UDS 0494.0 & $2.485_{2.965}^{2.005}$ & 182 & $0.07 \pm 0.02$ & $0.11 \pm 0.01$ & $0.34 \pm 0.05$ & $120 \pm 1$ & $0.7 \pm 0.1$ \\
\hline AS2UDS 0506.0 & $1.805_{2.095}^{1.555}$ & 123 & $0.06 \pm 0.02$ & $0.08 \pm 0.01$ & $0.69 \pm 0.03$ & $109 \pm 5$ & $0.3 \pm 0.1$ \\
\hline AS2UDS 0513.0 & $2.285_{2.515}^{2.025}$ & 91 & $0.03 \pm 0.01$ & $0.06 \pm 0.00$ & $0.26 \pm 0.03$ & $62 \pm 2$ & $1.0 \pm 0.2$ \\
\hline AS2UDS 0521.0 & $2.955_{3.225}^{2.565}$ & 331 & $0.06 \pm 0.02$ & $0.06 \pm 0.01$ & $0.90 \pm 0.03$ & $60 \pm 20$ & $0.2 \pm 0.3$ \\
\hline AS2UDS 0531.0 & $2.765_{2.945}^{2.515}$ & 407 & $0.06 \pm 0.02$ & $0.08 \pm 0.00$ & $0.60 \pm 0.03$ & $158 \pm 3$ & $0.5 \pm 0.1$ \\
\hline AS2UDS 0536.0 & $2.215_{2.481}^{1.949}$ & 269 & $0.09 \pm 0.02$ & $0.15 \pm 0.01$ & $0.36 \pm 0.06$ & $166 \pm 1$ & $1.1 \pm 0.1$ \\
\hline AS2UDS 0537.0 & $2.425_{3.035}^{1.795}$ & 372 & $0.10 \pm 0.02$ & $0.11 \pm 0.01$ & $0.77 \pm 0.04$ & $129 \pm 6$ & $0.3 \pm 0.1$ \\
\hline
\end{tabular}

